\title{
Archimedean Copula-Based Hesitant Fuzzy Information Aggregation Operators for Multiple Attribute Decision Making
}

\author{
Ju Wu, ${ }^{1,2}$ Lianming Mou, ${ }^{1,2}$ Fang Liu $\mathbb{D}^{1,2}$ Haobin Liu, ${ }^{1,2}$ and Yi Liu $\mathbb{D}^{1,2,3}$ \\ ${ }^{1}$ Data Recovery Key Laboratory of Sichuan Province, Neijiang Normal University, Neijiang 641000, Sichuan, China \\ ${ }^{2}$ School of Mathematics and Information Sciences, Neijiang Normal University, Neijiang 641000, Sichuan, China \\ ${ }^{3}$ Numerical Simulation Key Laboratory of Sichuan Province, Neijiang Normal University, Neijiang 641000, Sichuan, China
}

Correspondence should be addressed to Yi Liu; liuyiyl@126.com

Received 3 April 2020; Revised 2 June 2020; Accepted 9 June 2020; Published 3 July 2020

Academic Editor: Francesc Pozo

Copyright (@ $2020 \mathrm{Ju}$ Wu et al. This is an open access article distributed under the Creative Commons Attribution License, which permits unrestricted use, distribution, and reproduction in any medium, provided the original work is properly cited.

\begin{abstract}
In view of the good properties of copulas and their effective use in various fuzzy environments, the goal of the current study is to develop a series of aggregation operators for hesitant fuzzy information based on Archimedean copula and cocopula, which are applied to the MADM problems. Firstly, operational laws of hesitant fuzzy elements on the basis of copulas and cocopulas are defined which can show the relevance between hesitant fuzzy values. Secondly, four aggregation operators (AC-HFWA, ACGHFWA, AC-HFWG, and AC-GHFWG) under hesitant fuzzy environment are developed according to the proposed operational laws. The properties of these operators are also studied in detail, including idempotence, monotonicity, boundedness, etc. Subsequently, five special cases of copula are also given and the special forms of aggregation operator are obtained. In the end, an example is used to illustrate the application of the proposed approach in MADM problems. The influences of different generated functions and parameters are shown, and the feasibility of the proposed method is validated through comparative analyses.
\end{abstract}

\section{Introduction}

Multiple attribute decision making (MADM), also known as limited scheme multiobjective decision, is to select the optimal alternatives or ranking decision making problems in the case of considering multiple attributes. It is a vital part of modern decision science; its theories and methods have been widely utilized in engineering, technology, economy, management, military, and many other fields. One of the most important tasks of MADM is to fuse the attribute values given to each alternative by the decision maker and then summarize the decision maker's opinion on each alternative. In this process, a primary issue is to describe the values of criteria. For this issue, many experts proposed to adopt fuzzy sets. MADM problems with different kinds of fuzzy information are handled by utilizing fuzzy set (FS) [1] which is proposed by Zadeh and their various extensions, including the intuitionistic fuzzy set (IFS) [2], interval-valued intuitionistic fuzzy set (IVIFS) [3], hesitant fuzzy set (HFS) [4, 5], Pythagorean fuzzy set (PFS) [6], neutrosophic set (NS) [7], and so on.
In the numerous extensions of the FS, IFS as one of the most important, was introduced by Atanassov [2]. Because it provides a membership degree (MD), a nonmembership degree (NMD), and a hesitancy degree (HD) to each element, IFS is better at handling uncertainty and vagueness than FS. Since its emergence, IFS has attracted more and more researchers' attention. However, when giving the membership degree of an element, the difficulty of establishing the membership degree is not because we have a margin of error or some possibility distribution on the possibility values but because we have several possible values. For such cases, Torra and Narukawa [4] proposed hesitant fuzzy set (HFS) and indicated that the envelope of a hesitant fuzzy element (HFE) is an intuitionistic fuzzy value (IFV). So, all the operations on IFS can be suitable for HFS, and many research studies of IFS can be extended to HFS.

The aggregation operator, which fuses multiple information sources, plays a key role in the realization of collective opinions in MADM. In order to deal with information in different fuzzy environments, various 
aggregation operators are proposed. Weighted average (WA) operator and weighted geometry (WG) operator are the most commonly used integration operators in classical decision science theory. In the process of MADM, they have been deeply studied by scholars [8-12], which have been extended to the integration of different types of decision information, such as ordered weighted averaging operator (OWA) and ordered weighted geometry operator (OWG). Based on the defined operations for IFS, Xia and $\mathrm{Xu}$ [13] presented eight hesitant fuzzy aggregation operators, such as hesitant fuzzy weighted averaging (HFWA) operator, hesitant fuzzy weighted geometric (HFWG) operator, and so on. According to the operators mentioned above, many scholars investigated many operators to solve MCDM problems under hesitant fuzzy environment [14-21]. Qin et al. [22] developed some hesitant fuzzy aggregation operators based on Frank operations, such as HFFWA operator, HFFOWA operator, and so on. Yu et al. [23] studied a set of hesitant fuzzy Einstein aggregation operators, such as HFECOA operator, HFECOG operator, HFEPWA operator, and HFEPWG operator. Using the technique of obtaining values in the interval, Du et al. [24] proposed the generalized hesitant fuzzy harmonic mean operators including GHFWHM operator, GHFOWHM operator, and GHFHHM operator. Li and Chen [25] presented two new aggregation operators: belief structure hesitant fuzzy induced ordered weighted averaging operator and belief structure hesitant fuzzy induced ordered weighted geometric operator. Although the research and application of the integration operator have been well developed, the decision problem based on the integration operator has certain complexity, so it is necessary to conduct in-depth research on it and explore new information integration methods.

In the aforementioned aggregation operators under hesitant fuzzy environment, the operational laws of any two HFEs are built on the t-norms (TCs) and t-conorms (TCs). Commonly, TNs are applied to integrate MD of fuzzy sets, while copulas are tools to deal with probability distributions. Besides, there exist also TNs which are copulas and vice versa. Thus, the application of copulas in fuzzy sets has important practical significance. Copulas [26] can not only reveal the dependence among attributes but also prevent information loss in the midst of aggregation. There are two distinguishing features of copula: (1) copulas and cocopulas are flexible because decision makers can select different types of copulas and cocopulas to define the operations under fuzzy environment, and the results obtained from these operations are closed; (2) copula functions are flexible to capture the correlations among attributes in MADMs. Based on the two obvious characteristic, copulas have been applied to some MADMs. In the light of Archimedean copula, Tao et al. [27] studied a new computational model for unbalanced linguistic variables. Chen et al. [28] defined new aggregation operators in linguistic neutrosophic set based on copula and applied them to settle MCDM problems.

In this paper, based on the current research, the copulas are generalized to the HFS, and two kinds of hesitating fuzzy information integration operators based on copulas are proposed, which are applied to the MADM problems. For the goals, the structure of this work is arranged as follows. Some notions on hesitant fuzzy set and copulas are reviewed firstly in Section 2. The hesitant fuzzy weighted averaging operator-based Archimedean copulas (AC-HFWA) are defined in Section 3; before AC-HFWA is given, the operations of hesitant fuzzy elements based on Archimedean copula are also defined. After AC-HFWA is given, the generalized hesitant fuzzy weighted averaging operatorbased Archimedean copulas (AC-GHFWA) are introduced, and the properties of AC-HFWA and AC-HFWG are investigated along with the different cases. The hesitant fuzzy weighted geometry operator-based Archimedean copulas (AC-HFWG) are defined in Section 4; before AC-HFWG is given, the operations of hesitant fuzzy elements based on Archimedean copula are also defined. After AC-HFWG is given, the generalized hesitant fuzzy weighted geometry operator-based Archimedean copulas (AC-GHFWG) are introduced, and the properties of AC-HFWA and ACHFWG are investigated along with the different cases. In Section 5, the algorithm of MADM with hesitant fuzzy information based on AC-HFWA/AC-HFWG is constructed firstly; next, case analysis will be carried out and some comparisons with existing approaches in the hesitant fuzzy environment and merits of the proposed MADM approach based on AC-HFWA/AC-HFWG operators are analysed, and the conclusion will be obtained in Section 6 .

\section{Preliminaries}

In this section, we will retrospect the related concepts of HFS and copula and cocopula; these notions are the basis of this work.

\subsection{Hesitant Fuzzy Sets}

Definition 1 (see [5]). Let $S$ be a finite reference set. A hesitant fuzzy set $G$ on $S$ in terms of a function when applied to $S$ returns a subset of $[0,1]$ denoted by

$$
G=\{\langle s, g(h)\rangle \mid \forall s \in S\},
$$

where $g(h)$ is a collection of numbers $h_{i}$ from $[0,1]$, indicating the possible membership degrees of $\forall s \in S$ to $G$. We call $g(h)$ a hesitant fuzzy element (HFE) and $G$ the set of all HFEs.

To compare the HFEs, the comparison laws are defined as follows [5].

Definition 2 (see [5]). For a HFE $g(h)=\cup_{i=1}^{\natural g}\left\{h_{i}\right\}, \mu(g)=$ $(1 / \hbar g) \sum_{i=1}^{\natural g} h_{i}$ is called the score function of $g(h)$, where $h g$ is the number of possible elements in $g(h)$.

For two HFEs $g_{1}(h)$ and $g_{2}(h)$,

$$
\begin{aligned}
& \text { If } \mu\left(g_{1}\right)>\mu\left(g_{2}\right) \text {, then } g_{1}>g_{2} \text {; } \\
& \text { If } \mu\left(g_{1}\right)=\mu\left(g_{2}\right) \text {, then } g_{1}=g_{2} \text {. }
\end{aligned}
$$

\subsection{Copulas and Cocopulas}

Definition 3 (see [26]). A two-dimensional function $\Omega:[0,1]^{2} \longrightarrow[0,1]$ is called a copula, if the following conditions are met: 
(1) $\Omega(m, 1)=\Omega(1, m)=m, \Omega(m, 0)=\Omega(0, m)=0$

(2) $\Omega\left(m_{1}, n_{1}\right)-\Omega\left(m_{2}, n_{1}\right)-\Omega\left(m_{1}, n_{2}\right)+\Omega\left(m_{2}, n_{2}\right) \geq 0$

where $m, m_{1}, m_{2}, n_{1}, n_{2} \in[0,1]$ and $m_{1} \leq m_{2}, n_{1} \leq n_{2}$.

Definition 4 (see [29]). A copula $\Omega$ is named as an Archimedean copula, if there is a strictly decreasing and continuous function $\varsigma(\delta):[0,1] \longrightarrow[0, \infty]$ with $\varsigma(1)=0$, and $\sigma$ from $[0, \infty]$ to $[0,1]$ is defined as follows:

$$
\sigma(\delta)= \begin{cases}\varsigma^{-1}(\delta), & \delta \in[0, \varsigma(0)] ; \\ 0, & \delta \in[\varsigma(0),+\infty) .\end{cases}
$$

For all $(\delta, \varepsilon) \in[0,1]^{2}$, we have

$$
\sigma(\delta, \varepsilon)=\sigma(\varsigma(\delta)+\varsigma(\varepsilon))
$$

If $\Omega$ is strictly increasing on $[0,1]^{2}, \varsigma(0)=+\infty$, and $\sigma$ coincides with $\varsigma^{-1}$ on $[0,+\infty]$, then $\Omega$ is written as [30]

$$
\Omega(\delta, \varepsilon)=\varsigma^{-1}(\varsigma(\delta)+\varsigma(\varepsilon)),
$$

and the function $\varsigma$ is called a strict generator and $\Omega$ is called a strict Archimedean copula.

Definition 5 (see [31]). Let $\Omega$ be a copula, and the cocopula is introduced as follows:

$$
\Omega^{*}(\delta, \varepsilon)=1-\Omega(1-\delta, 1-\varepsilon) .
$$
be

If $\Omega$ is a strict Archimedean copula, $\Omega^{*}$ is also changed to

$\Omega^{*}(\delta, \varepsilon)=1-\Omega(1-\delta, 1-\varepsilon)=1-\varsigma^{-1}(\varsigma(1-\delta)+\varsigma(1-\varepsilon))$.

In order to introduce some new operations based on copulas and cocopulas mentioned above, the following conclusion is given firstly.

Theorem 1. For $\forall \delta, \varepsilon \in[0,1]$, then $0 \leq \Omega(\delta, \varepsilon) \leq 1,0 \leq$ $\Omega^{*}(\delta, \varepsilon) \leq 1$.

Proof. If $0 \leq \delta \leq \varepsilon \leq 1$, then $0 \leq 1-\varepsilon \leq 1-\delta \leq 1$. As $\varsigma$ is strictly decreasing and $\varsigma(1)=0, \varsigma(0)=+\infty$,

$$
\begin{aligned}
& 0 \leq \varsigma(\varepsilon) \leq \varsigma(\delta) \leq+\infty, \\
& 0 \leq \varsigma(1-\delta) \leq \varsigma(1-\varepsilon) \leq+\infty .
\end{aligned}
$$

$$
\begin{aligned}
& \text { So, } \\
& \qquad \begin{aligned}
\varsigma(\delta) & \leq \varsigma(\delta)+\varsigma(\varepsilon) \leq 2 \varsigma(\delta) \leq+\infty \\
\varsigma(1-\varepsilon) & \leq \varsigma(1-\delta)+\varsigma(1-\varepsilon) \leq 2 \varsigma(1-\varepsilon) \leq+\infty .
\end{aligned}
\end{aligned}
$$

We have

$0 \leq \varsigma^{-1}(\varsigma(\delta)+\varsigma(\varepsilon)) \leq \delta \leq \varepsilon \leq 1-\varsigma^{-1}(\varsigma(1-\delta)+\varsigma(1-\varepsilon)) \leq 1$.

Thus, Theorem 1 holds.

Definition 6. Let $\delta, \varepsilon \in[0,1]$; the algebra operations based on copula and cocopula are defined as follows:

$$
\begin{aligned}
& \text { (1) } \delta \oplus \varepsilon=\Omega^{*}(\delta, \varepsilon)=1-\varsigma^{-1}(\varsigma(1-\delta)+\varsigma(1-\varepsilon)), \\
& (2) \delta \otimes \varepsilon=\Omega(\delta, \varepsilon)=\varsigma^{-1}(\varsigma(\delta)+\varsigma(\varepsilon)) .
\end{aligned}
$$

It is easy to verify that $\oplus$ and $\otimes$ satisfy associative law, that is, for $\forall \delta, \varepsilon, v \in[0,1]$,

$$
\begin{aligned}
& (\delta \oplus \varepsilon) \oplus \nu=\delta \oplus(\varepsilon \oplus \nu), \\
& (\delta \otimes \varepsilon) \otimes \nu=\delta \otimes(\varepsilon \otimes \nu) .
\end{aligned}
$$

Theorem 2. For $\forall \delta \in[0,1], \rho \geq 0$, we have $\rho \delta=1-\varsigma^{-1}$ $(\rho \varsigma(1-\delta)), \delta^{\rho}=\varsigma^{-1}(\rho \varsigma(\delta))$.

\section{Archimedean Copula-Based Hesitant Fuzzy Weighted Averaging Operator (AC-HFWA)}

In this part, we will put forward the Archimedean copulabased HF weighted averaging operator (AC-HFWA). Before AC-HFWA is introduced, the new operations of HFE based on copula will be defined, and then some properties of ACHFWA are also investigated.

3.1. New Operations for HFEs Based on Copulas. We will give a new version of operational rules based on copulas and cocopulas.

Definition 7. Let $g_{1}(h)=\cup_{m_{1}=1}^{\natural g_{1}}\left\{h_{1 m_{1}}\right\}, g_{2}(h)=\cup_{m_{2}=1}^{\natural g_{2}}\left\{h_{2 m_{2}}\right\}$, and $g(h)=\cup_{i=1}^{\natural g}\left\{h_{i}\right\}$ be three HFEs and $\rho \geq 0$; the novel operational rules of HFEs are given as follows:

$$
\begin{aligned}
& g_{1} \oplus g_{2}=\bigcup_{\substack{h_{1 m_{1}} \in g_{1} \\
h_{2 m_{2}} \in g_{2}}}^{\bigcup}\left\{1-\varsigma^{-1}\left(\varsigma\left(1-h_{1 m_{1}}\right)+\varsigma\left(1-h_{2 m_{2}}\right)\right) \mid m_{1}=1,2, \ldots, \downarrow g_{1}, m_{2}=1,2, \ldots, \hbar g_{2}\right\}, \\
& g_{1} \otimes g_{2}=\bigcup_{h_{1 m_{1}} \in g_{1}}\left\{\varsigma^{-1}\left(\varsigma\left(h_{1 m_{1}}\right)+\varsigma\left(h_{2 m_{2}}\right)\right) \mid m_{1}=1,2, \ldots, \hbar g_{1}, m_{2}=1,2, \ldots, \hbar g_{2}\right\}, \\
& h_{2 m_{2}} \in g_{2} \\
& \rho g=\bigcup_{h_{i} \in g}\left\{1-\varsigma^{-1}\left(\rho \varsigma\left(1-h_{i}\right)\right) \mid i=1,2, \ldots, \curvearrowleft g\right\} \text {, } \\
& g^{\rho}=\bigcup_{h_{i} \in g}\left\{\varsigma^{-1}\left(\rho \varsigma\left(h_{i}\right)\right) \mid i=1,2, \ldots, \curvearrowleft g\right\} .
\end{aligned}
$$


From the above definition, the following conclusions can be easily drawn.

Theorem 3. Let $g_{1}, g_{2}$, and $g_{3}$ be three HFEs and $a, b, c \in R^{+}$; then, we have

$$
\begin{aligned}
& \text { (1) } g_{1} \oplus g_{2}=g_{2} \oplus g_{1}, \\
& \text { (2) }\left(g_{1} \oplus g_{2}\right) \oplus g_{3}=g_{1} \oplus\left(g_{2} \oplus g_{3}\right), \\
& \text { (3) } a g_{1} \oplus b g_{1}=(a+b) g_{1}, \\
& \text { (4) } a\left(b g_{1} \oplus c g_{2}\right)=a b g_{1} \oplus a c g_{2}, \\
& \text { (5) } a\left(b g_{1}\right)=a b g_{1}, \\
& \text { (6) } g_{1} \otimes g_{2}=g_{2} \otimes g_{1}, \\
& \text { (7) }\left(g_{1} \otimes g_{2}\right) \otimes g_{3}=g_{1} \otimes\left(g_{2} \otimes g_{3}\right) .
\end{aligned}
$$

The algorithms can be used to fuse the HF information and investigate their ideal properties which is the focus of the following sections.
3.2. AC-HFWA. In this section, the AC-HFWA will be introduced and the proposed operations of HFEs based on copula as well as the properties of AC-HFWA are investigated.

Definition 8. Let $G=\left\{g_{1}, g_{2}, \ldots, g_{n}\right\}$ be a set of $n$ HFEs and $\Phi$ be a function on $G, \Phi:[0,1]^{n} \longrightarrow[0,1]$; then, $\Phi(G)=\cup\left\{\Phi\left(g_{1}, g_{2}, \ldots, g_{n}\right)\right\}$.

Definition 9. Let $g_{i}(h)=\cup_{m_{i}=1}^{\natural g_{i}}\left\{h_{i m_{i}} \mid i=1,2, \ldots, n\right\}, \omega_{i}$ be the weight vector of $g_{i}$ with $\omega_{i} \in[0,1]$ and $\sum_{i=1}^{n} \omega_{i}=1$. Archimedean copula-based hesitant fuzzy weighted averaging operator (AC-HFWA) is defined as follows:

$$
\operatorname{AC}-\operatorname{HFWA}\left(g_{1}, g_{2}, \ldots, g_{n}\right)=\omega_{1} g_{1} \oplus \omega_{2} g_{2} \oplus \cdots \oplus \omega_{n} g_{n} \text {. }
$$

Theorem 4. Let $g_{i}(h)=\cup_{m_{i}=1}^{\natural g_{i}}\left\{h_{i m_{i}} \mid i=1,2, \ldots, n\right\}, \omega_{i}$ be the weight vector of $g_{i}$ with $\omega_{i} \in[0,1]$ and $\sum_{i=1}^{n} \omega_{i}=1$; then,

$$
\operatorname{AC}-\operatorname{HFWA}\left(g_{1}, g_{2}, \ldots, g_{n}\right)=\bigoplus_{i=1}^{n} \omega_{i} g_{i}=\bigcup_{h_{i m_{i}} \in g_{i}}\left\{1-\tau^{-1}\left(\sum_{i=1}^{n} \omega_{i} \tau\left(1-h_{i m_{i}}\right)\right) \mid m_{i}=1,2, \ldots, n g_{i}\right\}
$$

Proof. For $n=2$, we have

$$
\begin{aligned}
\operatorname{AC}-\operatorname{HFWA}\left(g_{1}, g_{2}\right)= & \omega_{1} g_{1} \oplus \omega_{2} g_{2} \\
= & \bigcup_{\substack{h_{1 m_{1}} \in g_{1} \\
h_{2 m_{2}} \in g_{2}}}\left\{1-\varsigma^{-1}\left(\omega_{1} \varsigma\left(1-h_{1 m_{1}}\right)+\omega_{2} \varsigma\left(1-h_{2 m_{2}}\right)\right) \mid m_{1}=1,2, \ldots, \pitchfork g_{1}, m_{2}=1,2, \ldots, \curvearrowleft g_{2}\right\}
\end{aligned}
$$

Suppose that equation (15) holds for $n=k$, that is,

Then,

$$
\begin{aligned}
& \operatorname{AC}-\operatorname{HFWA}\left(g_{1}, g_{2}, \ldots, g_{k}\right)=\omega_{1} g_{1} \oplus \omega_{2} g_{2} \oplus \cdots \oplus \omega_{k} g_{k} \\
& =\bigcup_{h_{i m_{i}} \in g_{i}}\left\{1-\tau^{-1}\left(\sum_{i=1}^{k} \omega_{i} \tau\left(1-h_{i m_{i}}\right)\right) \mid m_{i}=1,2, \ldots, \hbar g_{i}\right\} .
\end{aligned}
$$

$$
\begin{aligned}
\operatorname{AC}-\operatorname{HFWA}\left(g_{1}, g_{2}, \ldots, g_{k}, g_{k+1}\right)= & \bigoplus_{i=1}^{k} \omega_{i} g_{i} \oplus \omega_{k+1} g_{k+1} \\
= & \bigcup_{h_{i m_{i}} \in g_{i}}\left\{1-\tau^{-1}\left(\sum_{i=1}^{k} \omega_{i} \tau\left(1-h_{i m_{i}}\right)\right) \mid m_{i}=1,2, \ldots, \downarrow g_{i}\right\} \\
& \oplus \bigcup_{h_{k+1, m_{k}+1} \in g_{k+1}}\left\{1-\tau^{-1}\left(\omega_{k+1} \tau\left(1-h_{k+1, m_{k}+1}\right)\right) \mid m_{k+1}=1,2, \ldots, \downarrow g_{k+1}\right\} \\
= & \bigcup_{h_{i m_{i}} \in g_{i}}\left\{1-\tau^{-1}\left(\sum_{i=1}^{k} \omega_{i} \tau\left(1-h_{i m_{i}}\right)+\omega_{k+1} \tau\left(1-h_{k+1, m_{k}+1}\right)\right) \mid m_{i}=1,2, \ldots, n g_{i}\right\} \\
= & \bigcup_{h_{i m_{i}} \in g_{i}}\left\{1-\tau^{-1}\left(\sum_{i=1}^{k+1} \omega_{i} \tau\left(1-h_{i m_{i}}\right)\right) \mid m_{i}=1,2, \ldots, n g_{i}\right\} .
\end{aligned}
$$


Equation (15) holds for $n=k+1$. Thus, equation (15) holds for all $n$.

Theorem 5. Let $g_{i}(h)=\cup_{m_{i}=1}^{\natural g_{i}}\left\{h_{i m_{i}} \mid i=1,2, \ldots, n\right\}, \omega_{i}$ be the weight vector of $g_{i}$ with $\omega_{i} \in[0,1]$ and $\sum_{i=1}^{n} \omega_{i}=1$; then,

(1) (Idempotency) If $g_{1}=g_{2}=\cdots g_{n}=\{h\}, A C-H F W A$ $\left(g_{1}, g_{2}, \ldots, g_{n}\right)=\{h\}$.

(2) (Monotonicity) Let $g_{i}^{*}(h)=\cup_{m_{i}=1}^{\natural g_{i}^{*}}\left\{h_{i m_{i}}^{*} \mid i=1,2, \ldots\right.$, $n\}$; if $h_{i m_{i}} \leq h_{i m_{i}}^{*}$,

$\operatorname{AC}-\operatorname{HFWA}\left(g_{1}, g_{2}, \ldots, g_{n}\right) \leq \operatorname{AC}-\operatorname{HFWA}\left(g_{1}^{*}, g_{2}^{*}, \ldots, g_{n}^{*}\right)$.

(3) (Boundedness) If $h^{-}=\min _{i=1,2, \ldots, n}\left\{h_{i m_{i}}\right\}$ and $h^{+}=$ $\max _{i=1,2, \ldots, n}\left\{h_{i m_{i}}\right\}$,

$$
h^{-} \leq \operatorname{AC}-\operatorname{HFWA}\left(g_{1}, g_{2}, \ldots, g_{n}\right) \leq h^{+} \text {. }
$$

Proof (1) AC $-\operatorname{HFWA}\left(g_{1}, g_{2}, \ldots, g_{n}\right)=$

$\cup\left\{1-\varsigma^{-1}\left(\sum_{i=1}^{n} \omega_{i} \varsigma(1-h)\right) \mid h \in g_{i}, i=1,2, \ldots, n\right\}=\{h\}$.

(2) If $h_{i m_{i}} \leq h_{i m_{i}}^{*}, \quad \varsigma\left(1-h_{i m_{i}}\right) \leq \varsigma\left(1-h_{i m_{i}}^{*}\right)$ and $\sum_{i=1}^{n}$ $\omega_{i} \varsigma\left(1-h_{i m_{i}}\right) \leq \sum_{i=1}^{n} \omega_{i} \varsigma\left(1-h_{i m_{i}}^{*}\right)$.

Then, $\quad \varsigma^{-1}\left(\sum_{i=1}^{n} \omega_{i} \varsigma\left(1-h_{i m_{i}}\right)\right) \geq \varsigma^{-1}\left(\sum_{i=1}^{n} \omega_{i} \varsigma(1-\right.$ $\left.\left.h_{i m_{i}}^{*}\right)\right) \quad$ and $\quad 1-\varsigma^{-1}\left(\sum_{i=1}^{n} \omega_{i} \varsigma\left(1-h_{i m_{i}}\right)\right) \leq 1-\varsigma^{-1}$ $\left(\sum_{i=1}^{n} \omega_{i} \varsigma\left(1-h_{i m_{i}}^{*}\right)\right)$.

So, $\quad$ AC $-\operatorname{HFWA}\left(g_{1}, g_{2}, \ldots, g_{n}\right) \leq \mathrm{AC}-\mathrm{HFWA}$ $\left(g_{1}^{*}, g_{2}^{*}, \ldots, g_{n}^{*}\right)$.

(3) Suppose $h^{-}=\min _{i=1,2, \ldots, n}\left\{h_{i m_{i}}\right\} \quad$ and $\quad h^{+}=$ $\max _{i=1,2, \ldots, n}\left\{h_{i m_{i}}\right\}$.

Therefore, $1-h^{+} \leq 1-h_{i m_{i}}, 1-h^{-} \geq 1-h_{i m_{i}}$, for all $i$ and $m_{i}$.
Since $\varsigma$ is strictly decreasing, $\varsigma^{-1}$ is also strictly decreasing.

Then, $\quad \varsigma\left(1-h^{-}\right) \leq \varsigma\left(1-h_{i m_{i}}\right) \leq \varsigma\left(1-h^{+}\right), \forall i=1,2, \ldots$, $n$, and so

$$
\sum_{i=1}^{n} \omega_{i} \varsigma\left(1-h^{-}\right) \leq \sum_{i=1}^{n} \omega_{i} \varsigma\left(1-h_{i m_{i}}\right) \leq \sum_{i=1}^{n} \omega_{i} \varsigma\left(1-h^{+}\right)
$$

That is, $\varsigma\left(1-h^{-}\right) \leq \sum_{i=1}^{n} \omega_{i} \varsigma\left(1-h_{i m_{i}}\right) \leq \varsigma\left(1-h^{+}\right)$.

Therefore, $\quad \varsigma^{-1}\left(\varsigma\left(1-h^{+}\right)\right) \leq \varsigma^{-1}\left(\sum_{i=1}^{n} \omega_{i} \varsigma\left(1-h_{i m_{i}}\right)\right) \leq$ $\varsigma^{-1}\left(\varsigma\left(1-h^{-}\right)\right), h_{0} \leq 1-\varsigma^{-1}\left(\sum_{i=1}^{n} \omega_{i} \varsigma\left(1-h_{i m_{i}}\right)\right) \leq h^{+}$.

Definition 10. Let $g_{i}(h)=\cup_{m_{i}=1}^{\natural g_{i}}\left\{h_{i m_{i}} \mid i=1,2, \ldots, n\right\}, \omega_{i}$ be the weight vector of $g_{i}$ with $\omega_{i} \in[0,1]$ and $\sum_{i=1}^{n} \omega_{i}=1$. The Archimedean copula-based generalized hesitant fuzzy averaging operator (AC-GHFWA) is given by

$$
\begin{aligned}
\mathrm{AC} & -\operatorname{GHFWA}_{\theta}\left(g_{1}, g_{2}, \ldots, g_{n}\right) \\
& =\left(\omega_{1} g_{1}^{\theta} \oplus \omega_{2} g_{2}^{\theta} \oplus \cdots \oplus \omega_{n} g_{n}^{\theta}\right)^{1 / \theta}=\left(\bigoplus_{i=1}^{n} \omega_{i} g_{i}^{\theta}\right)^{1 / \theta} .
\end{aligned}
$$

Especially, when $\theta=1$, the AC-GHFWA operator becomes the AC-HFWA operator.

The following theorems are easily obtained from Theorem 4 and the operations of HFEs.

Theorem 6. Let $g_{i}(h)=\cup_{m_{i}=1}^{\natural g_{i}}\left\{h_{i m_{i}} \mid i=1,2, \ldots, n\right\}, \omega_{i}$ be the weight vector of $g_{i}$ with $\omega_{i} \in[0,1]$ and $\sum_{i=1}^{n} \omega_{i}=1$; then,

$$
\mathrm{AC}-\operatorname{GHFWA}_{\theta}\left(g_{1}, g_{2}, \ldots, g_{n}\right)=\bigcup_{h_{i m_{i}} \in g_{i}}\left\{-\varsigma^{-1}\left(\frac{1}{\theta}\left(\varsigma\left(1-\varsigma^{-1}\left(\sum_{i=1}^{n} \omega_{i}\left(\varsigma\left(1-\varsigma^{-1}\left(\theta \varsigma\left(h_{i m_{i}}\right)\right)\right)\right)\right)\right)\right)\right) \mid m_{i}=1,2, \ldots, \downarrow g_{i}\right\}
$$

Similar to Theorem 5, the properties of AC-GHFWA can be obtained easily.

Theorem 7. Let $g_{i}(h)=\cup_{m_{i}=1}^{\natural g_{i}}\left\{h_{i m_{i}} \mid i=1,2, \ldots, n\right\}, \omega_{i}$ be the weight vector of $g_{i}$ with $\omega_{i} \in[0,1]$ and $\sum_{i=1}^{n} \omega_{i}=1$; then,

(1) (Idempotency) If $g_{1}=g_{2}=\cdots g_{n}=\{h\}, \quad A C-$ $\operatorname{GHFWA}_{\theta}\left(g_{1}, g_{2}, \ldots, g_{n}\right)=\{h\}$.

(2) (Monotonicity) Let $g_{i}^{*}(h)=\cup_{m_{i}=1}^{\natural g_{i}^{*}}\left\{h_{i m_{i}}^{*} \mid i=\right.$ $1,2, \ldots, n\} ;$ if $h_{i m_{i}} \leq h_{i m_{i}}^{*}$,

$$
\begin{aligned}
& \mathrm{AC}-\mathrm{GHFWA}_{\theta}\left(g_{1}, g_{2}, \ldots, g_{n}\right) \\
& \quad \leq \mathrm{AC}-\mathrm{GHFWA}_{\theta}\left(g_{1}^{*}, g_{2}^{*}, \ldots, g_{n}^{*}\right) .
\end{aligned}
$$

(3) (Boundedness) If $h^{-}=\min _{i=1,2, \ldots, n}\left\{h_{i m_{i}}\right\}$ and $h^{+}=\max _{i=1,2, \ldots, n}\left\{h_{i m_{i}}\right\}$,

$$
h^{-} \leq \mathrm{AC}-\mathrm{GHFWA}_{\theta}\left(g_{1}, g_{2}, \ldots, g_{n}\right) \leq h^{+}
$$

3.3. Different Forms of AC-HFWA. We can see from Theorem 4 that some specific AC-HFWAs can be obtained when $\zeta$ is assigned different generators.

Case 1. If $\varsigma(t)=(-\ln t)^{\kappa}, \kappa \geq 1$, then $\varsigma^{-1}(t)=e^{-t^{1 / \kappa}}$. So, $\delta \oplus \varepsilon=1-e^{-\left((-\ln (1-\delta))^{\kappa}+\left((-\ln (1-\varepsilon))^{\kappa}\right)^{1 / \kappa}\right.}$, $\delta \otimes \varepsilon=1-e^{-\left((-\ln (\delta))^{\kappa}+\left((-\ln (\varepsilon))^{\kappa}\right)^{1 / \kappa}\right.}$. 


$$
\operatorname{AC}-\operatorname{HFWA}\left(g_{1}, g_{2}, \ldots, g_{n}\right)=\bigcup_{h_{i m_{i}} \in g_{i}}\left\{1-e^{-\left(\sum_{i=1}^{n} \omega_{i}\left(-\ln \left(1-h_{i m_{i}}\right)\right)^{\lambda}\right)^{1 / \lambda}} \mid m_{i}=1,2, \ldots, n g_{i}\right\}
$$

Specifically, when $\quad \kappa=1, \varsigma(t)=-\ln t$, then $\delta \oplus \varepsilon=1-(1-\delta)(1-\varepsilon), \quad \delta \otimes \varepsilon=\delta \varepsilon$, and the ACHFWA becomes the following:

$$
\begin{aligned}
\operatorname{HFWA}\left(g_{1}, g_{2}, \ldots, g_{n}\right) & =\bigcup_{h_{i m_{i}} \in g_{i}}\left\{1-\prod_{i=1}^{n}\left(1-h_{i m_{i}}\right)^{\omega_{i}} \mid m_{i}=1,2, \ldots, n g_{i}\right\}, \\
\operatorname{GHFWA}_{\theta}\left(g_{1}, g_{2}, \ldots, g_{n}\right) & =\bigcup_{h_{i m_{i}} \in g_{i}}\left\{\left(1-\prod_{i=1}^{n}\left(1-h_{i m_{i}}\right)^{\omega_{i}}\right)^{1 / \theta} \mid m_{i}=1,2, \ldots, \sharp g_{i}\right\} .
\end{aligned}
$$

They are the HF operators defined by Xia and Xu [13].

Case 2. If $\varsigma(t)=t^{-\kappa}-1, \kappa>0$, then $\varsigma^{-1}(t)=$ $(t+1)^{-(1 / \kappa)}$. So, $\quad \delta \oplus \varepsilon=1-\left((1-\delta)^{-\kappa}+(1-\varepsilon)^{-\kappa}\right.$ $-1)^{-1 / \kappa}, \delta \otimes \mathcal{E}=\left(\delta^{-\kappa}+\varepsilon^{-} \kappa-1\right)^{-1 / \kappa}$.

$$
\operatorname{AC}-\operatorname{HFWA}\left(g_{1}, g_{2}, \ldots, g_{n}\right)=\bigcup_{h_{i m_{i}} \in g_{i}}\left\{1-\left(\sum_{i=1}^{n} \omega_{i}\left(1-h_{i m_{i}}\right)^{-\kappa}\right)^{-1 / \kappa} \mid m_{i}=1,2, \ldots, n g_{i}\right\}
$$

Case 3. If $\varsigma(t)=-\ln \left(\left(e^{-\kappa t}-1\right) /\left(e^{-\kappa}-1\right)\right), \kappa \neq 0$, then $\varsigma^{-1}(t)=-(1 / \kappa) \ln \left(e^{-t}\left(e^{-\kappa}-1\right)+1\right)$. So, $\delta \oplus \varepsilon=1+$

$(1 / \kappa) \ln \left(\left(\left(e^{-\kappa(1-\delta)}-1\right)\left(e^{-\kappa(1-\varepsilon)}-1\right) /\left(e^{-\kappa}-1\right)\right)+1\right)$, $\delta \otimes \mathcal{E}=-(1 / \kappa) \ln \left(\left(\left(e^{-\kappa \delta}-1\right)\left(e^{-\kappa \varepsilon}-1\right) /\left(e^{-\kappa}-1\right)\right)+1\right)$.

$$
\operatorname{AC}-\operatorname{HFWA}\left(g_{1}, g_{2}, \ldots, g_{n}\right)=\bigcup_{h_{i m_{i}} \in g_{i}}\left\{1+\frac{1}{\kappa} \ln \left(\prod_{i=1}^{n}\left(e^{-\kappa\left(1-h_{i m_{i}}\right)}-1\right)^{\omega_{i}}+1\right) \mid m_{i}=1,2, \ldots, n g_{i}\right\} .
$$

Case 4. If $\varsigma(t)=\ln ((1-\kappa(1-t)) / t),-1 \leq \kappa<1$, then $\varsigma^{-1}(t)=\left((1-\kappa) /\left(e^{t}-\kappa\right)\right)$. So, $\delta \oplus \varepsilon=1-((1-\delta)$ $(1-\varepsilon) /(1-\kappa \delta \varepsilon)), \delta \otimes \varepsilon=(\delta \varepsilon /(1-\kappa(1-\delta)(1-\varepsilon)))$.

$$
\operatorname{AC}-\operatorname{HFWA}\left(g_{1}, g_{2}, \ldots, g_{n}\right)=\bigcup_{h_{i m_{i}} \in g_{i}}\left\{\frac{\prod_{i=1}^{n}\left(1-\kappa h_{i m_{i}}\right)^{\omega_{i}}-\prod_{i=1}^{n}\left(1-h_{i m_{i}}\right)^{\omega_{i}}}{\prod_{i=1}^{n}\left(1-\kappa h_{i m_{i}}\right)^{\omega_{i}}-\kappa \prod_{i=1}^{n}\left(1-h_{i m_{i}}\right)^{\omega_{i}}} \mid m_{i}=1,2, \ldots, \curvearrowleft g_{i}\right\}
$$

Case 5. If $\varsigma(t)=-\ln \left(1-(1-t)^{\kappa}\right), \kappa \geq 1$, then $\varsigma^{-1}(t)=1-\left(1-e^{-t}\right)^{1 / \kappa}$. So $\delta \oplus \varepsilon=\left(\delta^{\kappa}+\varepsilon^{\kappa}-\delta^{\kappa} \varepsilon^{\kappa}\right)^{1 / \kappa}$, $\delta \otimes \mathcal{E}=1-\left((1-\delta)^{\kappa}+(1-\varepsilon)^{\kappa}-(1-\delta)^{\kappa}(1-\varepsilon)^{\kappa}\right)^{1 / \kappa}$.

$$
\operatorname{AC}-\operatorname{HFWA}\left(g_{1}, g_{2}, \ldots, g_{n}\right)=\bigcup_{h_{i m_{i}} \in g_{i}}\left\{\left(1-\prod_{i=1}^{n}\left(1-h_{i m_{i}}^{\kappa}\right)^{\omega_{i}}\right)^{1 / \kappa} \mid m_{i}=1,2, \ldots, n g_{i}\right\} .
$$




\section{Archimedean Copula-Based Hesitant Fuzzy Weighted Geometric Operator (AC-HFWG)}

In this section, the Archimedean copula-based hesitant fuzzy weighted geometric operator (AC-HFWG) will be introduced, and some special forms of AC-HFWG operators will be discussed when the generator $\varsigma$ takes different functions.

4.1. AC-HFWG
Definition 11. Let $g_{i}(h)=\cup_{m_{i}=1}^{\natural g_{i}}\left\{h_{i m_{i}} \mid i=1,2, \ldots, n\right\}, \omega_{i}$ be the weight vector of $g_{i}$ with $\omega_{i} \in[0,1]$ and $\sum_{i=1}^{n} \omega_{i}=1$. The Archimedean copula-based hesitant fuzzy weighted geometric operator (AC-HFWG) is defined as follows:

$$
\operatorname{AC}-\operatorname{HFWG}\left(g_{1}, g_{2}, \ldots, g_{n}\right)=g_{1}^{\omega_{1}} \otimes g_{2}^{\omega_{2}} \otimes \cdots \otimes g_{n}^{\omega_{n}} .
$$

Theorem 8. Let $g_{i}(h)=\cup_{m_{i}=1}^{\natural g_{i}}\left\{h_{i m_{i}} \mid i=1,2, \ldots, n\right\}, \omega_{i}$ be the weight vector of $g_{i}$ with $\omega_{i} \in[0,1]$ and $\sum_{i=1}^{n} \omega_{i}=1$; then,

$$
\operatorname{AC}-\operatorname{HFWG}\left(g_{1}, g_{2}, \ldots, g_{n}\right)=\underset{h_{i m_{i}} \in g_{i}}{\cup}\left\{\varsigma^{-1}\left(\sum_{i=1}^{n} \omega_{i} \varsigma\left(h_{i m_{i}}\right)\right) \mid m_{i}=1,2, \ldots, n g_{i}\right\} .
$$

Proof. For $n=2$, we have

$$
\begin{aligned}
\operatorname{AC} & -\operatorname{HFWG}\left(g_{1}, g_{2}\right)=g_{1}^{\omega_{1}} \otimes g_{2}^{\omega_{2}} \\
& =\bigcup_{h_{1 m_{1}} \in g_{1} h_{2 m_{2}} \in g_{2}}\left\{\varsigma^{-1}\left(\omega_{1} \varsigma\left(h_{1 m_{1}}\right)+\omega_{2} \varsigma\left(h_{2 m_{2}}\right)\right) \mid m_{1}=1,2, \ldots, \pitchfork g_{1}, m_{2}=1,2, \ldots, \downarrow g_{2}\right\} .
\end{aligned}
$$

Suppose that equation (33) holds for $n=k$, that is,

$$
\operatorname{AC}-\operatorname{HFWG}\left(g_{1}, g_{2}, \ldots, g_{k}\right)=\bigcup_{h_{i m_{i}} \in g_{i}}\left\{\varsigma^{-1}\left(\sum_{i=1}^{k} \omega_{i} \varsigma\left(h_{i m_{i}}\right)\right) \mid m_{i}=1,2, \ldots, \downarrow g_{i}\right\}
$$

Then,

$$
\operatorname{AC}-\operatorname{HFWG}\left(g_{1}, g_{2}, \ldots, g_{k}, g_{k}+1\right)=\otimes_{i=1}^{k} g_{i}^{\omega_{i}} \oplus g_{k+1}^{\omega_{k+1}}=\underset{h_{i m_{i}} \in g_{i}}{\cup}\left\{\varsigma^{-1}\left(\sum_{i=1}^{k+1} \omega_{i} \varsigma\left(h_{i m_{i}}\right)\right) \mid m_{i}=1,2, \ldots, \downarrow g_{i}\right\}
$$

Equation (33) holds for $n=k+1$. Thus, equation (33) holds for all $n$.

Theorem 9. Let $g_{i}(h)=\cup_{m_{i}=1}^{\natural g_{i}}\left\{h_{i m_{i}} \mid i=1,2, \ldots, n\right\}, \omega_{i}$ be the weight vector of $g_{i}$ with $\omega_{i} \in[0,1]$ and $\sum_{i=1}^{n} \omega_{i}=1$; then,
(1) If $g_{1}=g_{2}=\cdots g_{n}=\{h\}, \quad A C-\operatorname{HFWG}\left(g_{1}, g_{2}\right.$, $\left.\ldots, g_{n}\right)=\{h\}$.

(2) Let $g_{i}^{*}(h)=\cup_{m_{i}=1}^{\natural g_{i}^{*}}\left\{h_{i m_{i}}^{*} \mid i=1,2, \ldots, n\right\}$; if $h_{i m_{i}} \leq h_{i m_{i}}^{*}$,

$$
\operatorname{AC}-\operatorname{HFWG}\left(g_{1}, g_{2}, \ldots, g_{n}\right) \leq \operatorname{AC}-\operatorname{HFWG}\left(g_{1}^{*}, g_{2}^{*}, \ldots, g_{n}^{*}\right)
$$

(3) If $h^{-}=\min _{i=1,2, \ldots, n}\left\{h_{i m_{i}}\right\}$ and $h^{+}=\max _{i=1,2, \ldots, n}\left\{h_{i m_{i}}\right\}$,

$$
h^{-} \leq \mathrm{AC}-\operatorname{HFWG}\left(g_{1}, g_{2}, \ldots, g_{n}\right) \leq h^{+}
$$

Proof. Suppose $h^{-}=\min _{i=1,2, \ldots, n}\left\{h_{i m_{i}}\right\}$ and $h^{+}=$ $\max _{i=1,2, \ldots, n}\left\{h_{i m_{i}}\right\}$.

Since $\varsigma$ is strictly decreasing, $\varsigma^{-1}$ is also strictly decreasing. 
Then, $\varsigma\left(h^{+}\right) \leq \varsigma\left(h_{i m_{i}}\right) \leq \varsigma\left(h^{-}\right), \forall i=1,2, \ldots, n$.

$\sum_{i=1}^{n} \omega_{i} \varsigma\left(h^{+}\right) \leq \sum_{i=1}^{n} \omega_{i} \varsigma\left(h_{i m_{i}}\right) \leq \sum_{i=1}^{n} \omega_{i} \varsigma\left(h^{-}\right), \quad \varsigma\left(h^{+}\right) \leq$ $\sum_{i=1}^{n} \omega_{i} \varsigma\left(h_{i m_{i}}\right) \leq \varsigma\left(h^{-}\right), \quad$ so $\quad \varsigma^{-1}\left(\varsigma\left(h^{-}\right)\right) \leq \varsigma^{-1}\left(\sum_{i=1}^{n}\right.$ $\left.\omega_{i} \varsigma\left(h_{i m_{i}}\right)\right) \leq \varsigma^{-1}\left(\varsigma\left(h^{+}\right)\right) h^{-} \leq \varsigma^{-1}\left(\sum_{i=1}^{n} \omega_{i} \varsigma\left(h_{i m_{i}}\right)\right) \leq h^{+}$.
Definition 12. Let $g_{i}(h)=\cup_{m_{i}=1}^{\natural g_{i}}\left\{h_{i m_{i}} \mid i=1,2, \ldots, n\right\}, \omega_{i}$ be the weight vector of $g_{i}$ with $\omega_{i} \in[0,1]$ and $\sum_{i=1}^{n} \omega_{i}=1$; then, the generalized hesitant fuzzy weighted geometric operator based on Archimedean copulas (AC-CHFWG) is defined as follows:

$$
\mathrm{AC}-\mathrm{GHFWG}_{\theta}\left(g_{1}, g_{2}, \ldots, g_{n}\right)=\frac{1}{\theta}\left(\left(\theta g_{1}\right)^{\omega_{1}} \otimes\left(\theta g_{2}\right)^{\omega_{2}} \otimes \cdots \otimes\left(\theta g_{n}\right)^{\omega_{n}}\right)=\frac{1}{\theta}\left(\bigotimes_{i=1}^{n}\left(\theta g_{i}\right)^{\omega_{i}}\right)
$$

Especially, when $\theta=1$, the AC-GHFWG operator becomes the AC-HFWG operator.
Theorem 10. Let $g_{i}(h)=\cup_{m_{i}=1}^{\natural g_{i}}\left\{h_{i m_{i}} \mid i=1,2, \ldots, n\right\}, \omega_{i}$ be the weight vector of $g_{i}$ with $\omega_{i} \in[0,1]$ and $\sum_{i=1}^{n} \omega_{i}=1$; then,

$$
\mathrm{AC}-\operatorname{GHFWG}_{\theta}\left(g_{1}, g_{2}, \ldots, g_{n}\right)=\bigcup_{h_{i m_{i}} \in g_{i}}\left\{1-\tau^{-1}\left(\frac{1}{\theta}\left(\tau\left(1-\tau^{-1}\left(\sum_{i=1}^{n} \omega_{i}\left(\tau\left(1-\tau^{-1}\left(\theta \tau\left(1-h_{i m_{i}}\right)\right)\right)\right)\right)\right)\right)\right) \mid m_{i}=1,2, \ldots, \mathfrak{\natural} g_{i}\right\} .
$$

Theorem 11. Let $g_{i}(h)=\cup_{m_{i}=1}^{\natural}\left\{h_{i m_{i}} \mid i=1,2, \ldots, n\right\}, \omega_{i}$ be the weight vector of $g_{i}$ with $\omega_{i} \in[0,1]$ and $\sum_{i=1}^{n} \omega_{i}=1$; then,
(2) Let $g_{i}^{*}(h)=\cup_{m_{i}=1}^{\natural g_{i}^{*}}\left\{h_{i m_{i}}^{*} \mid i=1,2, \ldots, n\right\} ;$ if $h_{i m_{i}} \leq h_{i m_{i}}^{*}$,

(1) If $g_{1}=g_{2}=\cdots g_{n}=\{h\}, A C-G H F W G_{\theta}\left(g_{1}, g_{2}, \ldots\right.$ ,$\left.g_{n}\right)=\{h\}$

$$
\mathrm{AC}-\mathrm{GHFWG}_{\theta}\left(g_{1}, g_{2}, \ldots, g_{n}\right) \leq \mathrm{AC}-\mathrm{GHFWG}_{\theta}\left(g_{1}^{*}, g_{2}^{*}, \ldots, g_{n}^{*}\right)
$$

(3) If $h^{-}=\min _{i=1,2, \ldots, n}\left\{h_{i m_{i}}\right\}$ and $h^{+}=\max _{i=1,2, \ldots, n}\left\{h_{i m_{i}}\right\}$,

$h^{-} \leq \mathrm{AC}-\mathrm{GHFWG}_{\theta}\left(g_{1}, g_{2}, \ldots, g_{n}\right) \leq h^{+}$.
4.2. Different Forms of AC-HFWG Operators. We can see from Theorem 8 that some specific AC-HFWGs can be obtained when $\varsigma$ is assigned different generators.

Case 1. If $\varsigma(t)=(-\ln t)^{\kappa}, \kappa \geq 1$, then $\varsigma^{-1}(t)=e^{-t^{1 / \kappa}}$. So, $\delta \oplus \varepsilon=1-e^{-\left((-\ln (1-\delta))^{\kappa}+\left((-\ln (1-\varepsilon))^{\kappa}\right)^{1 / k}\right.}$,

$\delta \otimes \varepsilon=e^{-\left((-\ln \delta)^{\kappa}+\left((-\ln \varepsilon)^{\kappa}\right)^{1 / \kappa}\right.}$.

$$
\operatorname{AC}-\operatorname{HFWG}\left(g_{1}, g_{2}, \ldots, g_{n}\right)=\bigcup_{h_{i m_{i}} \in g_{i}}\left\{e^{-\left(\sum_{i=1}^{n} \omega_{i}\left(-\ln h_{i m_{i}}\right)^{\kappa}\right)^{1 / \kappa}} \mid m_{i}=1,2, \ldots, n g_{i}\right\} .
$$

Specifically, when $\kappa=1$ and $\varsigma(t)=-\ln t$, then $\delta \oplus \varepsilon=$ $1-(1-\delta)(1-\varepsilon)$ and $\delta \otimes \varepsilon=\delta \varepsilon$, and the AC-HFWG

operator reduces to HFWG and GHFWG [13]:

$$
\begin{aligned}
\operatorname{HFWG}\left(g_{1}, g_{2}, \ldots, g_{n}\right) & =\underset{h_{i m_{i}} \in g_{i}}{\bigcup}\left\{\prod_{i=1}^{n} h_{i m_{i}}^{\omega_{i}} \mid m_{i}=1,2, \ldots, \curvearrowleft g_{i}\right\}, \\
\operatorname{GHFWG}_{\theta}\left(g_{1}, g_{2}, \ldots, g_{n}\right) & =\bigcup_{h_{i m_{i}} \in g_{i}}\left\{1-\left(1-\prod_{i=1}^{n}\left(1-\left(1-h_{i m_{i}}\right)^{\theta}\right)^{\omega_{i}}\right)^{1 / \theta} \mid m_{i}=1,2, \ldots, \curvearrowleft g_{i}\right\} .
\end{aligned}
$$


Case 2. If $\varsigma(t)=t^{-\kappa}-1, \kappa>0$, then $\varsigma^{-1}(t)=$ $(t+1)^{-(1 / \kappa)}$. So, $\quad \delta \oplus \varepsilon=1-\left((1-\delta)^{-\kappa}+(1-\varepsilon)^{-\kappa}\right.$ $-1)^{-(1 / \kappa)}, \delta \otimes \varepsilon=\left(\delta^{-\kappa}+\varepsilon^{-\kappa}-1\right)^{-1 / \kappa}$.

$$
\operatorname{AC}-\operatorname{HFWG}\left(g_{1}, g_{2}, \ldots, g_{n}\right)=\bigcup_{h_{i m_{i}} \in g_{i}}\left\{\left(\sum_{i=1}^{n} \omega_{i} h_{i m_{i}}^{-\kappa}\right)^{-(1 / \kappa)} \mid m_{i}=1,2, \ldots, \downarrow g_{i}\right\}
$$

Case 3. If $\varsigma(t)=-\ln \left(\left(e^{-\kappa t}-1\right) /\left(e^{-\kappa}-1\right)\right), \kappa \neq 0$, then $\varsigma^{-1}(t)=-(1 / \kappa) \ln \left(e^{-t}\left(e^{-\kappa}-1\right)+1\right)$. So, $\delta \oplus \varepsilon=1+$
$(1 / \kappa) \ln \left(\left(\left(e^{-\kappa(1-\delta)}-1\right)\left(e^{-\kappa(1-\varepsilon)}-1\right) /\left(e^{-\kappa}-1\right)\right)+1\right)$, $\delta \otimes \varepsilon=-(1 / k) \ln \left(\left(\left(e^{-\kappa \delta}-1\right)\left(e^{-\kappa \varepsilon}-1\right) /\left(e^{-\kappa}-1\right)\right)+1\right)$.

$$
\operatorname{AC}-\operatorname{HFWG}\left(g_{1}, g_{2}, \ldots, g_{n}\right)=\bigcup_{h_{i m_{i}} \in g_{i}}\left\{-\frac{1}{\kappa} \ln \left(\prod_{i=1}^{n}\left(e^{-\kappa h_{i m_{i}}}-1\right)^{\omega_{i}}+1\right) \mid m_{i}=1,2, \ldots, \downarrow g_{i}\right\} .
$$

Case 4. If $\varsigma(t)=\ln ((1-\kappa(1-t)) / t),-1 \leq \kappa<1$, then $\varsigma^{-1}(t)=\left((1-\kappa) /\left(e^{t}-\kappa\right)\right)$. So, $\quad \delta \oplus \varepsilon=1-((1-\delta)$ $(1-\varepsilon) /(1-\kappa \delta \varepsilon)), \delta \otimes \varepsilon=(\delta \varepsilon /(1-\kappa(1-\delta)(1-\varepsilon)))$.

$$
\operatorname{AC}-\operatorname{HFWG}\left(g_{1}, g_{2}, \ldots, g_{n}\right)=\bigcup_{h_{i m_{i}} \in g_{i}}\left\{\frac{(1-\kappa) \prod_{i=1}^{n} h_{i m_{i}}^{\omega_{i}}}{\prod_{i=1}^{n}\left(1-\kappa\left(1-h_{i m_{i}}\right)\right)^{\omega_{i}}-\kappa \prod_{i=1}^{n} h_{i m_{i}}^{\omega_{i}}} \mid m_{i}=1,2, \ldots, \curvearrowleft g_{i}\right\}
$$

Case 5. If $\varsigma(t)=-\ln \left(1-(1-t)^{\kappa}\right), \kappa \geq 1$, then $\varsigma^{-1}(t)=1-\left(1-e^{-t}\right)^{1 / \kappa}$. So, $\delta \oplus \varepsilon=\left(\delta^{\kappa}+\varepsilon^{\kappa}-\delta^{\kappa} \varepsilon^{\kappa}\right)^{1 / \kappa}$, $\delta \otimes \varepsilon=1-\left((1-\delta)^{\kappa}+(1-\varepsilon)^{\kappa}-(1-\delta)^{\kappa}(1-\varepsilon)^{\kappa}\right)^{1 / \kappa}$.

$$
\operatorname{AC}-\operatorname{HFWG}\left(g_{1}, g_{2}, \ldots, g_{n}\right)=\bigcup_{h_{i m_{i}} \in g_{i}}\left\{1-\left(1-\prod_{i=1}^{n}\left(1-\left(1-h_{i m_{i}}\right)^{\kappa}\right)^{\omega_{i}}\right)^{1 / \kappa} \mid m_{i}=1,2, \ldots, \downarrow g_{i}\right\} .
$$

4.3. The Properties of $A C-H F W A$ and $A C-H F W G$. It is seen from above discussion that AC-HFWA and AC-HFWG are functions with respect to the parameter which is from the generator $\varsigma$. In this section, we will introduce the properties of the AC-HFWA and AC-HFWG operator regarding to the parameter $\kappa$.

Theorem 12. Let $\varsigma(t)$ be the generator function of copula, and it takes five cases proposed in Section 4.2; then $\mu(A C-$
$\left.\operatorname{HFWA}\left(g_{1}, g_{2}, \ldots, g_{n}\right)\right)$ is an increasing function of $\kappa$, $\mu\left(A C-\operatorname{HFWG}\left(g_{1}, g_{2}, \ldots, g_{n}\right)\right)$ is an decreasing function of $\kappa$, and $\mu\left(A C-\operatorname{HFWA}\left(g_{1}, g_{2}, \ldots, g_{n}\right)\right) \geq \mu(A C-$ $\left.\operatorname{HFWG}\left(g_{1}, g_{2}, \ldots, g_{n}\right)\right)$.

Proof. (Case 1) When $\varsigma(t)=(-\ln t)^{\kappa}$ with $\kappa \geq 1$. By Definition 6, we have

$$
\begin{aligned}
& \mu(\mathrm{AC}-\operatorname{HFWA}(\kappa))=\frac{1}{\natural g_{1} \downarrow g_{2} \cdots \curvearrowleft g_{n}} \sum_{m_{1}=1}^{\natural g_{1}} \sum_{m_{2}=1}^{\natural g_{2}} \cdots \sum_{m_{n}=1}^{\natural g_{n}}\left(1-e^{-\left(\sum_{i=1}^{n} \omega_{i}\left(-\ln \left(1-h_{i m_{i}}\right)\right)^{\kappa}\right)^{1 / \kappa}}\right), \\
& \mu(\mathrm{AC}-\operatorname{HFWG}(\kappa))=\frac{1}{\natural g_{1} \downarrow g_{2} \cdots \downarrow g_{n}} \sum_{m_{1}=1}^{\natural g_{1}} \sum_{m_{2}=1}^{\natural g_{2}} \cdots \sum_{m_{n}=1}^{\natural g_{n}}\left(e^{-\left(\sum_{i=1}^{n} \omega_{i}\left(-\ln \left(1-h_{i m_{i}}\right)\right)^{\kappa}\right)^{1 / \kappa}}\right) .
\end{aligned}
$$


Suppose $1 \leq \kappa_{1}<\kappa_{2} ;$ according to reference [10], $\left(\sum_{i=1}^{n} \omega_{i} a_{i}^{\kappa}\right)^{1 / \kappa}$ is an increasing function of $\kappa$. So,

$$
\begin{gathered}
\left(\sum_{i=1}^{n} \omega_{i}\left(-\ln \left(1-h_{i m_{i}}\right)\right)^{\kappa_{1}}\right)^{1 / \kappa_{1}} \leq\left(\sum_{i=1}^{n} \omega_{i}\left(-\ln \left(1-h_{i m_{i}}\right)\right)^{\kappa_{2}}\right)^{1 / \kappa_{2}}, \\
\left(\sum_{i=1}^{n} \omega_{i}\left(-\ln h_{i m_{i}}\right)^{\kappa_{1}}\right)^{1 / \kappa_{1}} \leq\left(\sum_{i=1}^{n} \omega_{i}\left(-\ln h_{i m_{i}}\right)^{\kappa_{2}}\right)^{1 / \kappa_{2}} .
\end{gathered}
$$

Furthermore,

$$
\begin{gathered}
1-e^{-\left(\sum_{i=1}^{n} \omega_{i}\left(-\ln \left(1-h_{i m_{i}}\right)\right)^{\kappa_{1}}\right)^{1 / \kappa_{1}}} \leq 1-e^{-\left(\sum_{i=1}^{n} \omega_{i}\left(-\ln \left(1-h_{i m_{i}}\right)\right)^{\kappa_{2}}\right)^{1 / \kappa_{2}}}, \\
e^{-\left(\sum_{i=1}^{n} \omega_{i}\left(-\ln \left(1-h_{i m_{i}}\right)\right)^{\kappa_{1}}\right)^{1 / \kappa_{1}}} \geq e^{-\left(\sum_{i=1}^{n} \omega_{i}\left(-\ln \left(1-h_{i m_{i}}\right)\right)^{\kappa_{2}}\right)^{1 / \kappa_{2}}} .
\end{gathered}
$$

Therefore, $\mu\left(\mathrm{AC}-\operatorname{HFWA}\left(\kappa_{1}\right)\right) \leq \mu\left(\mathrm{AC}-\operatorname{HFWA}\left(\kappa_{2}\right)\right)$, $\mu\left(\mathrm{AC}-\operatorname{HFWG}\left(\kappa_{1}\right)\right) \geq \mu\left(\mathrm{AC}-\operatorname{HFWG}\left(\kappa_{2}\right)\right)$. Because $\kappa \geq 1$,

$$
\begin{aligned}
e^{-\left(\sum_{i=1}^{n} \omega_{i}\left(-\ln \left(1-h_{i m_{i}}\right)\right)^{\kappa}\right)^{1 / \kappa}}+e^{-\left(\sum_{i=1}^{n} \omega_{i}\left(-\ln h_{i m_{i}}\right)^{\kappa}\right)^{1 / \kappa}} & \leq e^{-\left(\sum_{i=1}^{n} \omega_{i}\left(-\ln \left(1-h_{i m_{i}}\right)\right)\right)}+e^{-\left(\sum_{i=1}^{n} \omega_{i}\left(-\ln h_{i m_{i}}\right)\right)} \\
& =\prod_{i=1}^{n}\left(1-h_{i m_{i}}\right)^{\omega_{i}}+\prod_{i=1}^{n} h_{i m_{i}}^{\omega_{i}} \leq \sum_{i=1}^{n} \omega_{i}\left(1-h_{i m_{i}}\right)+\sum \omega_{i} h_{i m_{i}}=\sum_{i=1}^{n} \omega_{i}=1 .
\end{aligned}
$$

So, $1-e^{-\left(\sum_{i=1}^{n} \omega_{i}\left(-\ln \left(1-h_{i m_{i}}\right)\right)^{\kappa}\right)^{1 / \kappa}} \geq e^{-\left(\sum_{i=1}^{n} \omega_{i}\left(-\ln h_{i m_{i}}\right)^{\kappa}\right)^{1 / \kappa}}$.

That is, $\mu\left(\mathrm{AC}-\operatorname{HFWA}\left(g_{1}, g_{2}, \ldots, g_{n}\right)\right) \geq \mu(\mathrm{AC}-$ $\left.\operatorname{HFWG}\left(g_{1}, g_{2}, \ldots, g_{n}\right)\right)$.
So Theorem 12 holds under Case 1.

Case 2. When $\varsigma(t)=t^{-\kappa}-1, \kappa>0$. Firstly,

$$
\begin{aligned}
& \mu(\mathrm{AC}-\operatorname{HFWA}(\kappa))=\frac{1}{\natural g_{1} \curvearrowleft g_{2} \cdots \curvearrowleft g_{n}} \sum_{m_{1}=1}^{\natural g_{1}} \sum_{m_{2}=1}^{\natural g_{2}} \cdots \sum_{m_{n}=1}^{\natural g_{n}}\left(1-\varsigma^{-1}\left(\sum_{i=1}^{n} \omega_{i} \varsigma\left(1-h_{i m_{i}}\right)\right)\right), \\
& \mu(\mathrm{AC}-\operatorname{HFWG}(\kappa))=\frac{1}{\natural g_{1} \curvearrowleft g_{2} \cdots \curvearrowleft g_{n}} \sum_{m_{1}=1}^{\natural g_{1}} \sum_{m_{2}=1}^{\natural g_{2}} \cdots \sum_{m_{n}=1}^{\natural g_{n}}\left(\varsigma^{-1}\left(\sum_{i=1}^{k} \omega_{i} \varsigma\left(h_{i m_{i}}\right)\right)\right) .
\end{aligned}
$$

Secondly, because $\varsigma(\kappa, t)=t^{-\kappa}-1, \kappa>0,0<t<1$, $(\partial \varsigma / \partial \kappa)=t^{-\kappa} \ln t, t^{-\kappa}>0, \ln t<0$, and then $(\partial \varsigma / \partial \kappa)<0$, $\varsigma(\kappa, t)$ is decreasing with respect to $\kappa$.

$\varsigma^{-1}(\kappa, t)=(t+1)^{-(1 / \kappa)}, \kappa>0,0<t<1$, so $\left(\partial \varsigma^{-1} / \partial \kappa\right)=$ $(t+1)^{-(1 / \kappa)} \ln (t+1)\left(1 / \kappa^{2}\right), \quad(t+1)^{-(1 / \kappa)}>0, \ln (t+1)>0$, $\left(1 / \kappa^{2}\right)>0$, and then $\left(\partial \varsigma^{-1} / \partial \kappa\right)>0, \varsigma^{-1}(\kappa, t)$ is decreasing with respect to $\kappa$.
Thus, $1-\varsigma^{-1}\left(\sum_{i=1}^{n} \omega_{i} \varsigma\left(1-h_{i m_{i}}\right)\right)$ is decreasing with respect to $\kappa$, and $\varsigma^{-1}\left(\sum_{i=1}^{k} \omega_{i} \varsigma\left(h_{i m_{i}}\right)\right)$ is decreasing with respect to $\kappa$. Suppose $1 \leq \kappa_{1}<\kappa_{2}$; we have $\mu\left(\mathrm{AC}-\operatorname{HFWA}\left(\kappa_{1}\right)\right) \leq \mu\left(\mathrm{AC}-\operatorname{HFWA}\left(\kappa_{2}\right)\right) \quad$ and $\mu\left(\mathrm{AC}-\operatorname{HFWG}\left(\kappa_{1}\right)\right) \geq \mu\left(\mathrm{AC}-\operatorname{HFWG}\left(\kappa_{2}\right)\right)$.

Lastly, 


$$
\begin{aligned}
\left(\sum_{i=1}^{n} \omega_{i}\left(1-h_{i m_{i}}\right)^{-\kappa}\right)^{-(1 / \kappa)}+\left(\sum_{i=1}^{n} \omega_{i} h_{i m_{i}}^{-\kappa}\right)^{-(1 / \kappa)} & <\lim _{\kappa \longrightarrow 0}\left(\sum_{i=1}^{n} \omega_{i}\left(1-h_{i m_{i}}\right)^{-\kappa}\right)^{-(1 / \kappa)}+\lim _{\kappa \longrightarrow 0}\left(\sum_{i=1}^{n} \omega_{i} h_{i m_{i}}^{-\kappa}\right)^{-(1 / \kappa)} \\
& =e^{\sum_{i=1}^{n} \omega_{i} \ln \left(1-h_{i m_{i}}\right)}+e^{\sum_{i=1}^{n} \omega_{i} \ln \left(h_{i m_{i}}\right)}=\prod_{i=1}^{n}\left(1-h_{i m_{i}}\right)^{\omega_{i}}+\prod_{i=1}^{n} h_{i m_{i}}^{\omega_{i}} \\
& \leq \sum_{i=1}^{n} \omega_{i}\left(1-h_{i m_{i}}\right)+\sum \omega_{i} h_{i m_{i}}=\sum_{i=1}^{n} \omega_{i}=1 .
\end{aligned}
$$

That is, Theorem 12 holds under Case 2.

Case 3. When $\varsigma(t)=-\ln \left(\left(e^{-\kappa t}-1\right) /\left(e^{-\kappa}-1\right)\right), \kappa \neq 0$. Firstly,

$$
\begin{aligned}
& \mu(\mathrm{AC}-\operatorname{HFWA}(\kappa))=\frac{1}{\natural g_{1} \curvearrowleft g_{2} \cdots \curvearrowleft g_{n}} \sum_{m_{1}=1}^{\natural g_{1}} \sum_{m_{2}=1}^{\natural g_{2}} \cdots \sum_{m_{n}=1}^{\natural g_{n}}\left(1-\varsigma^{-1}\left(\sum_{i=1}^{n} \omega_{i} \varsigma\left(1-h_{i m_{i}}\right)\right)\right), \\
& \mu(\operatorname{AC}-\operatorname{HFWG}(\kappa))=\frac{1}{\natural g_{1} \downarrow g_{2} \cdots \curvearrowleft g_{n}} \sum_{m_{1}=1}^{\natural g_{1}} \sum_{m_{2}=1}^{\natural g_{2}} \cdots \sum_{m_{n}=1}^{\natural g_{n}}\left(\varsigma^{-1}\left(\sum_{i=1}^{k} \omega_{i} \varsigma\left(h_{i m_{i}}\right)\right)\right) .
\end{aligned}
$$

Secondly, $\varsigma(\kappa, t)=-\ln \left(\left(e^{-\kappa t}-1\right) /\left(e^{-\kappa}-1\right)\right), \kappa \neq 0,0<t<1$.

If $\varsigma=-\ln \varsigma_{1}, \quad \varsigma_{1}=\left(\left(\varsigma_{2}^{t}-1\right) /\left(\varsigma_{2}-1\right)\right), \quad \varsigma_{2}=e^{-\kappa}, \kappa \neq 0$, $0<t<1$.

Because $\varsigma$ is decreasing with respect to $\varsigma_{1}, \varsigma_{1}$ is decreasing with respect to $\varsigma_{2}$, and $\varsigma_{2}$ is decreasing with respect to $\kappa$, $\varsigma(\kappa, t)$ is decreasing with respect to $\kappa$.

$$
\varsigma^{-1}(\kappa, t)=-\frac{1}{\kappa} \ln \left(e^{-t}\left(e^{-\kappa}-1\right)+1\right), \quad \kappa \neq 0,0<t<1 .
$$

Suppose $\quad \varsigma^{-1}=-\ln \psi_{1}, \quad \psi_{1}=\psi_{2}^{1 / \kappa}, \quad \psi_{2}=e^{-t} \psi_{3}+1$, $\varsigma_{3}=e^{-\kappa}-1, \kappa \neq 0,0<t<1$.
Because $\varsigma^{-1}$ is decreasing with respect to $\psi_{1}, \psi_{1}$ is decreasing with respect to $\psi_{2}$, and $\psi_{2}$ is decreasing with respect to $\psi_{3}, \psi_{3}$ is decreasing with respect to $\kappa$. Then, $\varsigma^{-1}(\kappa, t)$ is decreasing with respect to $\kappa$.

Thus, $1-\varsigma^{-1}\left(\sum_{i \overline{\bar{k}} 1}^{n} \omega_{i} \varsigma\left(1-h_{i m_{i}}\right)\right)$ is decreasing with respect to $\kappa$ and $\varsigma^{-1}\left(\sum_{i=1}^{k} \omega_{i} \varsigma\left(h_{i m_{i}}\right)\right)$ is decreasing with respect to $\kappa$.

Suppose $1 \leq \kappa_{1}<\kappa_{2}$.

Therefore, $\mu\left(\mathrm{AC}-\operatorname{HFWA}\left(\kappa_{1}\right)\right) \leq \mu\left(\mathrm{AC}-\operatorname{HFWA}\left(\kappa_{2}\right)\right)$ and $\mu\left(\operatorname{AC}-\operatorname{HFWG}\left(\kappa_{1}\right)\right) \geq \mu\left(\operatorname{AC}-\operatorname{HFWG}\left(\kappa_{2}\right)\right)$.

Lastly, when $-\propto<\kappa<+\propto$,

$$
\begin{aligned}
& -\frac{1}{\kappa} \ln \left(\prod_{i=1}^{n}\left(e^{-\kappa h_{i m_{i}}}-1\right)^{\omega_{i}}+1\right) \\
& \leq \lim _{\kappa \longrightarrow-\infty} \frac{\ln \left(\prod_{i=1}^{n}\left(e^{-\kappa h_{i m_{i}}}-1\right)^{\omega_{i}}+1\right)}{-\kappa}=\lim _{\kappa \longrightarrow-\infty} \frac{\left(\prod_{i=1}^{n}\left(e^{-\kappa h_{i m_{i}}}-1\right)^{\omega_{i}} /\left(\prod_{i=1}^{n}\left(e^{-\kappa h_{i m_{i}}}-1\right)^{\omega_{i}}+1\right)\right)\left(\sum _ { i = 1 } ^ { n } \omega _ { i } ( - h _ { i m _ { i } } ) \left(e^{\left.\left.-\kappa h_{i m_{i}} /\left(e^{-\kappa h_{i m_{i}}}-1\right)\right)\right)}-1\right.\right.}{-1} \\
& \quad \lim _{\kappa \longrightarrow-\propto}\left(\frac{\prod_{i=1}^{n}\left(e^{-\kappa h_{i m_{i}}}-1\right)^{\omega_{i}}}{\prod_{i=1}^{n}\left(e^{-\kappa h_{i m_{i}}}-1\right)^{\omega_{i}}+1}\right)\left(\sum_{i=1}^{n} \omega_{i} h_{i m_{i}} \frac{e^{-\kappa h_{i m_{i}}}}{e^{-\kappa h_{i m_{i}}}-1}\right)=\sum_{i=1}^{n} \omega_{i} h_{i m_{i}} \\
& \quad-\frac{1}{\kappa} \ln \left(\prod_{i=1}^{n}\left(e^{-\kappa\left(1-h_{i m_{i}}\right)}-1\right)^{\omega_{i}}+1\right) \leq \sum_{i=1}^{n} \omega_{i}\left(1-h_{i m_{i}}\right) .
\end{aligned}
$$

$$
\text { So, }-(1 / \kappa) \ln \left(\prod_{i=1}^{n}\left(e^{-} \quad \kappa\left(1-h_{i m_{i}}\right)-1\right)^{\omega_{i}}+1\right)-(1 / k)
$$
$\ln \left(\prod_{i=1}^{n}\left(e^{-\kappa h_{i m_{i}}}-1\right)^{\omega_{i}}+1\right) \leq \sum_{i=1}^{n} \omega_{i}\left(1-h_{i m_{i}}\right)+\sum_{i=1}^{n} \omega_{i} h_{i m_{i}}$ $=1$.
That is, Theorem 12 holds under Case 3.

Case 4. When $\varsigma(t)=\ln ((1-\kappa(1-t)) / t),-1 \leq \kappa<1$. Firstly, 


$$
\begin{aligned}
& \mu(\mathrm{AC}-\operatorname{HFWA}(\kappa))=\frac{1}{\natural g_{1} \natural g_{2} \cdots \curvearrowleft g_{n}} \sum_{m_{1}=1}^{\natural g_{1}} \sum_{m_{2}=1}^{\natural g_{2}} \cdots \sum_{m_{n}=1}^{\natural g_{n}}\left(1-\varsigma^{-1}\left(\sum_{i=1}^{n} \omega_{i} \varsigma\left(1-h_{i m_{i}}\right)\right)\right), \\
& \mu(\mathrm{AC}-\operatorname{HFWG}(\kappa))=\frac{1}{\natural g_{1} \llbracket g_{2} \cdots \natural g_{n}} \sum_{m_{1}=1}^{\natural g_{1}} \sum_{m_{2}=1}^{\natural g_{2}} \cdots \sum_{m_{n}=1}^{\natural g_{n}}\left(\varsigma^{-1}\left(\sum_{i=1}^{k} \omega_{i} \varsigma\left(h_{i m_{i}}\right)\right)\right) .
\end{aligned}
$$

Secondly, because $\quad \varsigma(\kappa, t)=\ln ((1-\kappa(1-t)) / t),-1$ $\leq \kappa<1,0<t<1$, so $\quad(\partial \varsigma / \partial \kappa)=(t(t-1) /(1-\kappa(1-t)))$, $t(t-1)<0$.

Suppose $\quad \varsigma_{1}(\kappa, t)=1-\kappa(1-t),-1 \leq \kappa<1,0<t<1$, $\left(\partial \varsigma_{1} / \partial \kappa\right)=t-1<0, \varsigma_{1}(\kappa, t)$ is decreasing with respect to $\kappa$.

So, $\varsigma_{1}(\kappa, t)>\varsigma_{1}(1, t)=t>0$; then, $(\partial \varsigma / \partial \kappa)<0$, and $\varsigma(\kappa, t)$ is decreasing with respect to $\kappa$.

As $\quad \varsigma^{-1}(\kappa, t)=(1-\kappa) /\left(e^{t}-\kappa\right),-1 \leq \kappa<1,0<t<1$, $\left(\partial \varsigma^{-1} / \partial \kappa\right)=\left(\left(e^{t}+1\right) /\left(e^{t}-\kappa\right)^{2}\right), \quad e^{t}+1>0, \quad\left(e^{t}-\kappa\right)^{2}>0 ;$ then $\left(\partial \varsigma^{-1} / \partial \kappa\right)>0, \varsigma^{-1}(\kappa, t)$ is decreasing with respect to $\kappa$. Thus, $1-\varsigma^{-1}\left(\sum_{i=1}^{n} \omega_{i} \varsigma\left(1-h_{i m_{i}}\right)\right)$ is decreasing with respect to $\kappa$ and $\varsigma^{-1}\left(\sum_{i=1}^{k} \omega_{i} \varsigma\left(h_{i m_{i}}\right)\right)$ is decreasing with respect to $\kappa$.

Suppose $1 \leq \kappa_{1}<\kappa_{2}$. We have $\mu\left(\mathrm{AC}-\operatorname{HFWA}\left(\kappa_{1}\right)\right)$ $\leq \mu\left(\mathrm{AC}-\operatorname{HFWA}\left(\kappa_{2}\right)\right)$ and $\mu\left(\mathrm{AC}-\operatorname{HFWG}\left(\kappa_{1}\right)\right) \geq \mu(\mathrm{AC}$ $\left.-\operatorname{HFWG}\left(\kappa_{2}\right)\right)$.

Lastly, because $y=x^{\omega_{i}}, \omega_{i} \in(0,1)$ is decreasing with respect to $\kappa,\left(1-h_{i}^{2}\right)^{\omega_{i}} \geq 1,\left(1-\left(1-h_{i}\right)^{2}\right)^{\omega_{i}} \geq 1$; then,

$$
\begin{array}{r}
\prod_{i=1}^{n}\left(1+h_{i m_{i}}\right)^{\omega_{i}}+\prod_{i=1}^{n}\left(1-h_{i m_{i}}\right)^{\omega_{i}} \geq 2\left(\prod_{i=1}^{n}\left(1+h_{i m_{i}}\right)^{\omega_{i}} \prod_{i=1}^{n}\left(1-h_{i m_{i}}\right)^{\omega_{i}}\right)^{1 / 2}=2\left(\prod_{i=1}^{n}\left(1-h_{i m_{i}}^{2}\right)^{\omega_{i}}\right)^{1 / 2} \geq 2 \\
\prod_{i=1}^{n}\left(2-h_{i m_{i}}\right)^{\omega_{i}}+\prod_{i=1}^{n} h_{i m_{i}}^{\omega_{i}} \geq 2\left(\prod_{i=1}^{n}\left(2-h_{i m_{i}}\right)^{\omega_{i}} \prod_{i=1}^{n} h_{i m_{i}}^{\omega_{i}}\right)^{1 / 2}=2\left(\prod_{i=1}^{n}\left(1-\left(1-h_{i m_{i}}\right)^{2}\right)^{\omega_{i}}\right)^{1 / 2} \geq 2 .
\end{array}
$$

When $-1 \leq \kappa<1$, we have

$$
\begin{aligned}
& \left(\frac{\prod_{i=1}^{n}\left(1-h_{i m_{i}}\right)^{\omega_{i}}-\kappa \prod_{i=1}^{n}\left(1-h_{i m_{i}}\right)^{\omega_{i}}}{\prod_{i=1}^{n}\left(1-\kappa h_{i m_{i}}\right)^{\omega_{i}}-\kappa \prod_{i=1}^{n}\left(1-h_{i m_{i}}\right)^{\omega_{i}}}\right)+\left(\frac{(1-\kappa) \prod_{i=1}^{n} h_{i m_{i}}^{\omega_{i}}}{\prod_{i=1}^{n}\left(1-\kappa\left(1-h_{i m_{i}}\right)\right)^{\omega_{i}}-\kappa \prod_{i=1}^{n} h_{i m_{i}}^{\omega_{i}}}\right) \\
& \quad \leq\left(\frac{\prod_{i=1}^{n}\left(1-h_{i m_{i}}\right)^{\omega_{i}}+\prod_{i=1}^{n}\left(1-h_{i m_{i}}\right)^{\omega_{i}}}{\prod_{i=1}^{n}\left(1+h_{i m_{i}}\right)^{\omega_{i}}+\prod_{i=1}^{n}\left(1-h_{i m_{i}}\right)^{\omega_{i}}}\right)+\left(\frac{2 \prod_{i=1}^{n} h_{i m_{i}}^{\omega_{i}}}{\prod_{i=1}^{n}\left(1+\left(1-h_{i m_{i}}\right)\right)^{\omega_{i}}+\prod_{i=1}^{n} h_{i m_{i}}^{\omega_{i}}}\right) \\
& \quad=\left(\frac{2 \prod_{i=1}^{n}\left(1-h_{i m_{i}}\right)^{\omega_{i}}}{\prod_{i=1}^{n}\left(1+h_{i m_{i}}\right)^{\omega_{i}}+\prod_{i=1}^{n}\left(1-h_{i m_{i}}\right)^{\omega_{i}}}\right)+\left(\frac{2 \prod_{i=1}^{n} h_{i m_{i}}^{\omega_{i}}}{\prod_{i=1}^{n}\left(2-h_{i m_{i}}\right)^{\omega_{i}}+\prod_{i=1}^{n} h_{i m_{i}}^{\omega_{i}}}\right) \\
& \quad \leq \prod_{i=1}^{n}\left(1-h_{i m_{i}}\right)^{\omega_{i}}+\prod_{i=1}^{n} h_{i m_{i}}^{\omega_{i}} \leq \sum_{i=1}^{n} \omega_{i}\left(1-h_{i m_{i}}\right)+\sum \omega_{i} h_{i m_{i}}=\sum_{i=1}^{n} \omega_{i}=1 .
\end{aligned}
$$

So,

$$
\frac{\prod_{i=1}^{n}\left(1-\kappa h_{i m_{i}}\right)^{\omega_{i}}-\prod_{i=1}^{n}\left(1-h_{i m_{i}}\right)^{\omega_{i}}}{\prod_{i=1}^{n}\left(1-\kappa h_{i m_{i}}\right)^{\omega_{i}}-\kappa \prod_{i=1}^{n}\left(1-h_{i m_{i}}\right)^{\omega_{i}}} \geq \frac{(1-\kappa) \prod_{i=1}^{n} h_{i m_{i}}^{\omega_{i}}}{\prod_{i=1}^{n}\left(1-\kappa\left(1-h_{i m_{i}}\right)\right)^{\omega_{i}}-\kappa \prod_{i=1}^{n} h_{i m_{i}}^{\omega_{i}}} .
$$


Therefore, $\quad \mu\left(\mathrm{AC}-\operatorname{HFWA}\left(g_{1}, g_{2}, \ldots, g_{n}\right)\right) \geq \mu(\mathrm{AC}-$ $\left.\operatorname{HFWG}\left(g_{1}, g_{2}, \ldots, g_{n}\right)\right)$. That is, Theorem 12 holds under Case 4.
Case 5. When $\varsigma(t)=-\ln \left(1-(1-t)^{\kappa}\right), \kappa \geq 1$.

Firstly,

$$
\begin{aligned}
& \mu(\operatorname{AC}-\operatorname{HFWA}(\kappa))=\frac{1}{\natural g_{1} \natural g_{2} \cdots \curvearrowleft g_{n}} \sum_{m_{1}=1}^{\natural g_{1}} \sum_{m_{2}=1}^{\natural g_{2}} \cdots \sum_{m_{n}=1}^{\natural g_{n}}\left(1-\varsigma^{-1}\left(\sum_{i=1}^{n} \omega_{i} \varsigma\left(1-h_{i m_{i}}\right)\right)\right), \\
& \mu(\mathrm{AC}-\operatorname{HFWG}(\kappa))=\frac{1}{\natural g_{1} \downarrow g_{2} \cdots \natural g_{n}} \sum_{m_{1}=1}^{\natural g_{1}} \sum_{m_{2}=1}^{\natural g_{2}} \cdots \sum_{m_{n}=1}^{\natural g_{n}}\left(\varsigma^{-1}\left(\sum_{i=1}^{k} \omega_{i} \varsigma\left(h_{i m_{i}}\right)\right)\right) .
\end{aligned}
$$

Secondly, because $\varsigma(\kappa, t)=-\ln \left(1-(1-t)^{\kappa}\right), \kappa \geq 1,0$ $<t<1, \quad(\partial \varsigma / \partial \kappa)=\left((1-t)^{\kappa} \ln (1-t) /\left(1-(1-t)^{\kappa}\right)\right)$, $\ln (1-t)<0$.

Suppose $\varsigma_{1}(\kappa, t)=(1-t)^{\kappa}, \kappa \geq 1,0<t<1, \quad\left(\partial \varsigma_{1} / \partial \kappa\right)=$ $(1-t)^{\kappa} \ln (1-t)<0, \varsigma_{1}(\kappa, t)$ is decreasing with respect to $\kappa$, so $0<\varsigma_{1}(\kappa, t) \leq \varsigma_{1}(1, t)=1-t<1,1-(1-t)^{\kappa}>0$.

Then, $(\partial \varsigma / \partial \kappa)<0, \varsigma(\kappa, t)$ is decreasing with respect to $\kappa$.

$\varsigma^{-1}(\kappa, t)=1-\left(1-e^{-t}\right)^{1 / \kappa}, \kappa \geq 1,0<t<1$, so $\left(\partial \varsigma^{-1} / \partial \kappa\right)$ $=\left(1-e^{-t}\right)^{1 / \kappa} \ln \left(1-e^{-t}\right)\left(-\left(1 / \kappa^{2}\right)\right), \quad\left(1-e^{-t}\right)^{1 / \kappa}>0$, $\ln \left(1-e^{-t}\right)<0,-\left(1 / \kappa^{2}\right)<0$; then, $\left(\partial \varsigma^{-1} / \partial \kappa\right)>0, \varsigma^{-1}(\kappa, t)$ is decreasing with respect to $\kappa$.

Thus, $1-\varsigma^{-1}\left(\sum_{i=1}^{n} \omega_{i} \varsigma\left(1-h_{i m_{i}}\right)\right)$ is decreasing with respect to $\kappa$ and $\varsigma^{-1}\left(\sum_{i=1}^{n} \omega_{i} \varsigma\left(h_{i m_{i}}\right)\right)$ is decreasing with respect to $\kappa$.

Lastly, suppose $1 \leq \kappa_{1}<\kappa_{2}$, Therefore, $\mu(\mathrm{AC}-\mathrm{HFWA}$ $\left.\left(\kappa_{1}\right)\right) \leq \mu\left(\mathrm{AC}-\operatorname{HFWA}\left(\kappa_{2}\right)\right), \mu\left(\mathrm{AC}-\operatorname{HFWG}\left(\kappa_{1}\right)\right) \geq \mu(\mathrm{AC}$ $\left.-\operatorname{HFWG}\left(\kappa_{2}\right)\right)$.

Because $\kappa \geq 1$,

$$
\begin{aligned}
& \left(\left(\prod_{i=1}^{n}\left(1-h_{i m_{i}}^{\kappa}\right)^{\omega_{i}}\right)^{1 / \kappa}\right)+\left(1-\left(1-\prod_{i=1}^{n}\left(1-\left(1-h_{i m_{i}}\right)^{\kappa}\right)^{\omega_{i}}\right)^{1 / \kappa}\right) \\
& \leq\left(\left(\prod_{i=1}^{n}\left(1-h_{i m_{i}}^{1}\right)^{\omega_{i}}\right)^{1}\right)+\left(1-\left(1-\prod_{i=1}^{n}\left(1-\left(1-h_{i m_{i}}\right)^{1}\right)^{\omega_{i}}\right)^{1}\right) \\
& =\prod_{i=1}^{n}\left(1-h_{i m_{i}}\right)^{\omega_{i}}+\prod_{i=1}^{n} h_{i m_{i}}^{\omega_{i}} \leq \sum_{i=1}^{n} \omega_{i}\left(1-h_{i m_{i}}\right)+\sum \omega_{i} h_{i m_{i}}=\sum_{i=1}^{n} \omega_{i}=1 .
\end{aligned}
$$

So, $\quad 1-e^{-\left(\sum_{i=1}^{n} \omega_{i}\left(-\ln \left(1-h_{i i_{i}}\right)\right)^{k}\right)^{1 / \kappa}} \geq e^{-\left(\left(\sum_{i=1}^{n} \omega_{i}\left(-\ln \left(h_{i m_{i}}\right)\right)^{\kappa}\right)\right)^{1 / \kappa}}$, $\mu\left(\operatorname{AC}-\operatorname{HFWA}\left(g_{1}, g_{2}, \ldots, g_{n}\right)\right) \geq \mu\left(\operatorname{AC}-\operatorname{HFWG}\left(g_{1}, g_{2}\right.\right.$, $\left.\left.\ldots, g_{n}\right)\right)$.

That is, Theorem 12 holds under Case 5.

\section{MADM Approach Based on AC-HFWA and AC-HFWG}

From the above analysis, a novel decision making way will be given to address MADM problems under HF environment based on the proposed operators.

Let $Y=\left\{\Psi_{i}(i=1,2, \ldots, m)\right\}$ be a collection of alternatives and $C=\left\{G_{j}(j=1,2, \ldots, n)\right\}$ be the set of attributes, whose weight vector is $W=\left(\omega_{1}, \omega_{2}, \ldots, \omega_{n}\right)^{T}$, satisfying $\omega_{j} \in[0,1]$ and $\sum_{j=1}^{n} \omega_{j}=1$. If DMs provide several values for the alternative $\Upsilon_{i}$ under the attribute $G_{j}(j=1,2, \ldots, n)$ with anonymity, these values can be considered as a HFE $h_{i j}$. In that case, if two DMs give the same value, then the value emerges only once in $h_{i j}$. In what follows, the specific algorithm for MADM problems under HF environment will be designed.
Step 1. The DMs provide their evaluations about the alternative $\Upsilon_{i}$ under the attribute $G_{j}$, denoted by the HFEs $h_{i j}, i=1,2, \ldots, m ; j=1,2, \ldots, n$.

Step 2. Use the proposed AC-HFWA (AC-HFWG) to fuse the HFE $y_{i}(i=1,2, \ldots, m)$ for $\Upsilon_{i}(i=1,2, \ldots, m)$. Step 3. The score values $\mu\left(y_{i}\right)(i=1,2, \ldots, m)$ of $y_{i}$ are calculated using Definition 6 and compared.

Step 4. The order of the attributes $\Upsilon_{i}(i=1,2, \ldots, m)$ is given by the size of $\mu\left(y_{i}\right)(i=1,2, \ldots, m)$.

5.1. Illustrative Example. An example of stock investment will be given to illustrate the application of the proposed method. Assume that there are four short-term stocks $\Upsilon_{1}, \Upsilon_{2}, \Upsilon_{3}, \Upsilon_{4}$. The following four attributes $\left(G_{1}, G_{2}, G_{3}, G_{4}\right)$ should be considered: $G_{1}$-net value of each share; $G_{2}$-earnings per share; $G_{3}$-capital reserve per share; and $G_{4}$-accounts receivable. Assume the weight vector of each attributes is $W=(0.2,0.3,0.15,0.35)^{T}$.

Next, we will use the developed method to find the ranking of the alternatives and the optimal choice. 
Step 1. The decision matrix is given by DM and is shown in Table 1.

Step 2. Use the AC-HFWA operator to fuse the HEE $y_{i}(i=1, \ldots, 4)$ for $\Upsilon_{i}(i=1, \ldots, 4)$.
Take $\Upsilon_{4}$ as an example, and let $\varsigma(t)=(-\ln t)^{1.2}$ in Case 1 ; we have

$$
\begin{aligned}
y_{4}= & \operatorname{AC}-\operatorname{HFWA}\left(g_{1}, g_{2}, g_{3}, g_{4}\right)=\oplus_{j=1}^{4} \omega_{j} g_{j}=\underset{h_{j m_{j}} \in g_{j}}{\cup}\left\{1-e^{-\left(\sum_{j=1}^{4} \omega_{i}\left(1-h_{j m_{j}}\right)^{1.2}\right)^{5 / 6}} \mid m_{j}=1,2, \ldots, \hbar g_{j}\right\} \\
= & \{0.5870,0.6139,0.6021,0.6278,0.6229,0.6470,0.7190,0.7361,0.7286,0.6117,0.6367,0.6257,0.6496,0.6450,0.6675, \\
& 0.7347,0.7508,0.7437,0.6303,0.6538,0.6435,0.6660,0.6617,0.6829,0.7466,0.7618,0.7551,0.7451,0.7419,0.7574, \\
& 0.7591,0.7561,0.7707,0.7697,0.7669,0.7808\} .
\end{aligned}
$$

Step 3. Compute the score values $\mu\left(y_{i}\right)(i=1, \ldots, 4)$ of $y_{i}(i=1, \ldots, 4)$ by Definition 6. $\mu\left(y_{4}\right)=0.6944$. Similarly,

$\mu\left(y_{1}\right)=0.5761, \mu\left(y_{2}\right)=0.6322, \mu\left(y_{3}\right)=0.5149$.

Step 4. Rank the alternatives $\Upsilon_{i}(i=1, \ldots, 4)$ and select the desirable one in terms of comparison rules. Since $\mu\left(y_{4}\right)>\mu\left(y_{2}\right)>\mu\left(y_{1}\right)>\mu\left(y_{3}\right)$, we can obtain the rank of alternatives as $\Upsilon_{4}>\Upsilon_{2}>\Upsilon_{1}>\Upsilon_{3}$, and $\Upsilon_{4}$ is the best alternative.

\subsection{Sensitivity Analysis}

5.2.1. The Effect of Parameters on the Results. We take Case 1 as an example to analyse the effect of parameter changes on the result. The results are shown in Table 2 and Figure 1.

It is easy to see from Table 2 that the scores of alternatives by the AC-HFWA operator increase as the value of the parameter $\kappa$ ranges from 1 to 5 . This is consistent with Theorem 12. But the order has not changed, and the best alternative is consistent.

5.2.2. The Effect of Different Types of Generators on the Results in AC-HFWA. The ordering results of alternatives using other generators proposed in present work are listed in Table 3. Figure 2 shows more intuitively how the score function varies with parameters.

The results show that the order of alternatives obtained by applying different generators and parameters is different, the ranking order of alternatives is almost the same, and the desirable one is always $\Upsilon_{4}$, which indicates that AC-HFWA operator is highly stable.

5.2.3. The Effect of Different Types of Generators on the Results in AC-HFWG. In step 2, if we utilize the AC-HFWG operator instead of the AC-HFWA operator to calculate the values of the alternatives, the results are shown in Table 4.

Figure 3 shows the variation of the values with parameter $\kappa$. We can find that the ranking of the alternatives may change when $\kappa$ changes in the AC-HFWG operator. With the increase in $\kappa$, the ranking results change from $\Upsilon_{4}>\Upsilon_{1}>\Upsilon_{3}>\Upsilon_{2}$ to $\Upsilon_{4}>\Upsilon_{3}>\Upsilon_{1}>\Upsilon_{2}$ and finally settle at $\Upsilon_{3}>\Upsilon_{4}>\Upsilon_{1}>\Upsilon_{2}$, which indicates that AC-HFWG has certain stability. In the decision process, DMs can confirm the value of $\kappa$ in accordance with their preferences.

Through the analysis of Tables 3 and 4, we can find that the score values calculated by the AC-HFWG operator decrease with the increase of parameter $\kappa$. For the same generator and parameter $\kappa$, the values obtained by the ACHFWA operator are always greater than those obtained by the AC-HFWG operator, which is consistent with Theorem 12.

5.2.4. The Effect of Parameter $\theta$ on the Results in AC-GHFWA and AC-GHFWG. We employ the AC-GHFWA operator and AC-GHFWG operator to aggregate the values of the alternatives, taking Case 1 as an example. The results are listed in Tables 5 and 6 . Figures 4 and 5 show the variation of the score function with $\kappa$ and $\theta$.

The results indicate that the scores of alternatives by the AC-GHFWA operator increase as the parameter $\kappa$ goes from 0 to 10 and the parameter $\theta$ ranges from 0 to 10 , and the ranking of alternatives has not changed. But the score values of alternatives reduce, and the order of alternatives obtained by the AC-GHFWG operator changes significantly.

5.3. Comparisons with Existing Approach. The above results indicate the effectiveness of our method, which can solve MADM problems. To further prove the validity of our method, this section compares the existing methods with our proposed method. The previous methods include hesitant fuzzy weighted averaging (HFWA) operator (or HFWG operator) by Xia and Xu [13], hesitant fuzzy Bonferroni means (HFBM) operator by Zhu and Xu [32], and hesitant fuzzy Frank weighted average (HFFWA) operator (or HFFWG operator) by Qin et al. [22]. Using the data in reference [22] and different operators, Table 7 is obtained. The order of these operators is $A_{6}>A_{2}>A_{3}>A_{5}>A_{1}>A_{4}$, except for the HFBM operator which is $A_{6}>A_{2}>$ $A_{3}>A_{1}>A_{5}>A_{4}$. 
TABle 1: HF decision matrix [13].

\begin{tabular}{lcccc}
\hline Alternatives & $G_{1}$ & $G_{2}$ & $G_{3}$ & $G_{4}$ \\
\hline$\Upsilon_{1}$ & $\{0.2,0.4,0.7\}$ & $\{0.2,0.6,0.8\}$ & $\{0.2,0.3,0.6,0.7,0.9\}$ & $\{0.3,0.4,0.5,0.7,0.8\}$ \\
$\Upsilon_{2}$ & $\{0.2,0.4,0.7,0.9\}$ & $\{0.1,0.2,0.4,0.5\}$ & $\{0.3,0.4,0.6,0.9\}$ & $\{0.5,0.6,0.8,0.9\}$ \\
$\Upsilon_{3}$ & $\{0.3,0.5,0.6,0.7\}$ & $\{0.2,0.4,0.5,0.6\}$ & $\{0.3,0.5,0.7,0.8\}$ & $\{0.2,0.5,0.6,0.7\}$ \\
$\Upsilon_{4}$ & $\{0.3,0.5,0.6\}$ & $\{0.2,0.4\}$ & $\{0.5,0.6,0.7\}$ & $\{0.8,0.9\}$ \\
\hline
\end{tabular}

TABLE 2: The ordering results obtained by AC-HFWA in Case 1.

\begin{tabular}{llllll}
\hline Parameter $\kappa$ & $\mu\left(\Upsilon_{1}\right)$ & $\mu\left(\Upsilon_{2}\right)$ & $\mu\left(\Upsilon_{3}\right)$ & $\mu\left(\Upsilon_{4}\right)$ & Ranking order \\
\hline 1 & 0.5612 & 0.6009 & 0.5178 & 0.6524 & $\Upsilon_{4}>\Upsilon_{2}>\Upsilon_{1}>\Upsilon_{3}$ \\
1.2 & 0.5720 & 0.6160 & 0.5249 & 0.6678 & $\Upsilon_{4}>\Upsilon_{2}>\Upsilon_{1}>\Upsilon_{3}$ \\
2 & 0.6084 & 0.6621 & 0.5498 & 0.7156 & $\Upsilon_{4}>\Upsilon_{2}>\Upsilon_{1}>\Upsilon_{3}$ \\
2.5 & 0.6258 & 0.6823 & 0.5626 & 0.7365 & $\Upsilon_{4}>\Upsilon_{2}>\Upsilon_{1}>\Upsilon_{3}$ \\
3 & 0.6399 & 0.6981 & 0.5736 & 0.7525 & $\Upsilon_{4}>\Upsilon_{2}>\Upsilon_{1}>\Upsilon_{3}$ \\
5 & 0.6757 & 0.7356 & 0.6045 & 0.7893 & $\Upsilon_{4}>\Upsilon_{2}>\Upsilon_{1}>\Upsilon_{3}$ \\
\hline
\end{tabular}

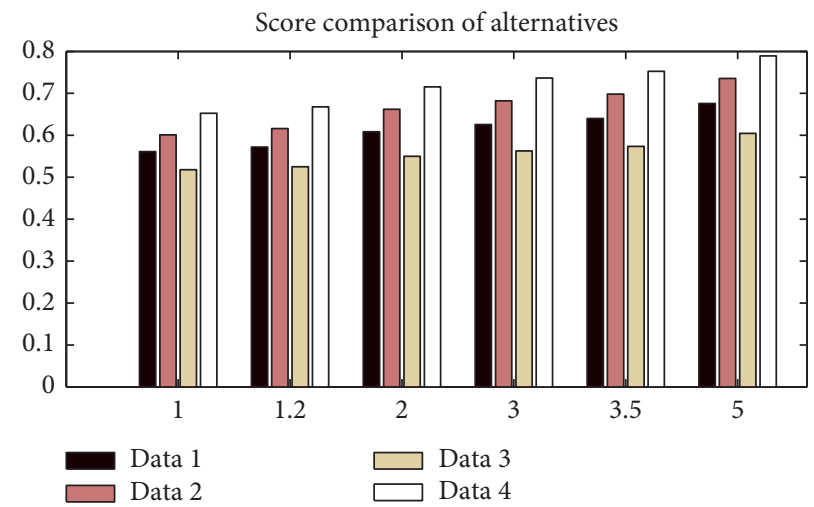

FIgUre 1: The score values obtained by AC-HFWA in Case 1.

TABLE 3: The ordering results obtained by AC-HFWA in Cases 2-5.

\begin{tabular}{lcccccc}
\hline Functions & Parameter $\lambda$ & $\mu\left(\Upsilon_{1}\right)$ & $\mu\left(\Upsilon_{2}\right)$ & $\mu\left(\Upsilon_{3}\right)$ & $\mu\left(\Upsilon_{4}\right)$ & Ranking order \\
\hline \multirow{3}{*}{ Case 2 } & 1 & 0.6059 & 0.6681 & 0.5425 & 0.7256 & $\Upsilon_{4}>\Upsilon_{2}>\Upsilon_{1}>\Upsilon_{3}$ \\
& 2 & 0.6411 & 0.7110 & 0.5656 & 0.7694 & $\Upsilon_{4}>\Upsilon_{2}>\Upsilon_{1}>\Upsilon_{3}$ \\
& 3 & 0.6650 & 0.7351 & 0.5851 & 0.7932 & $\Upsilon_{4}>\Upsilon_{2}>\Upsilon_{1}>\Upsilon_{3}$ \\
Case 3 & 1 & 0.5710 & 0.6143 & 0.5241 & 0.6665 & $\Upsilon_{4}>\Upsilon_{2}>\Upsilon_{1}>\Upsilon_{3}$ \\
& 2 & 0.5815 & 0.6278 & 0.5311 & 0.6806 & $\Upsilon_{4}>\Upsilon_{2}>\Upsilon_{1}>\Upsilon_{3}$ \\
\hline \multirow{3}{*}{ Case 4 } & 3 & 0.5922 & 0.6410 & 0.5386 & 0.6943 & $\Upsilon_{4}>\Upsilon_{2}>\Upsilon_{1}>\Upsilon_{3}$ \\
& 0.25 & 0.5666 & 0.6084 & 0.5212 & 0.6604 & $\Upsilon_{4}>\Upsilon_{2}>\Upsilon_{1}>\Upsilon_{3}$ \\
& 0.5 & 0.5739 & 0.6188 & 0.5256 & 0.6717 & $\Upsilon_{4}>\Upsilon_{2}>\Upsilon_{1}>\Upsilon_{3}$ \\
Case 5 & 0.75 & 0.5849 & 0.6349 & 0.5320 & 0.6894 & $\Upsilon_{4}>\Upsilon_{2}>\Upsilon_{1}>\Upsilon_{3}$ \\
& 1 & 0.5612 & 0.6009 & 0.5178 & 0.6524 & $\Upsilon_{4}>\Upsilon_{2}>\Upsilon_{1}>\Upsilon_{3}$ \\
& 2 & 0.5830 & 0.6279 & 0.5337 & 0.6781 & $\Upsilon_{4}>\Upsilon_{2}>\Upsilon_{1}>\Upsilon_{3}$ \\
\hline
\end{tabular}

(1) From the previous argument, it is obvious to find that Xia's operators [13] and Qin's operators [22] are special cases of our operators. When $\kappa=1$ in Case 1 of our proposed operators, AC-HFWA reduces to HFWA and AC-HFWG reduces to HFWG. When $\kappa=1$ in Case 3 of our proposed operators, ACHFWA becomes HFFWA and AC-HFWG becomes HFFWG. The operational rules of Xia's method are algebraic $\mathrm{t}$-norm and algebraic $\mathrm{t}$-conorm, and the operational rules of Qin's method are Frank t-norm and Frank t-conorm, which are all special forms of copula and cocopula. Therefore, our proposed operators are more general. Furthermore, the proposed approach will supply more choice for DMs in real MADM problems.

(2) The results of Zhu's operator [32] are basically the same as ours, but Zhu's calculations were more complicated. Although we all introduce parameters, 


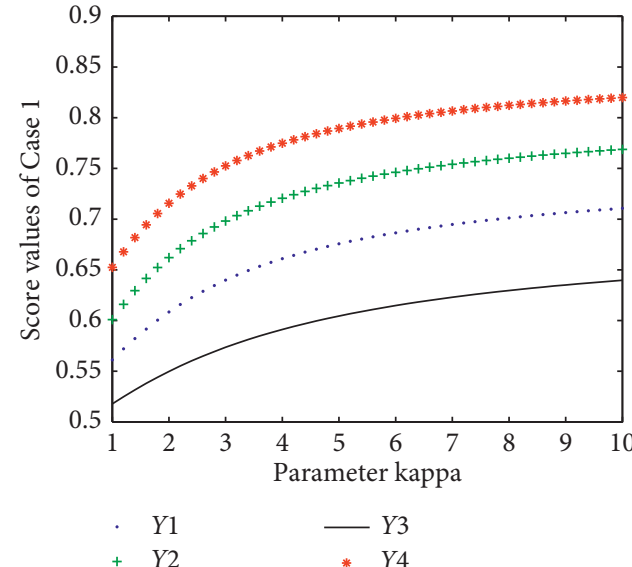

(a)

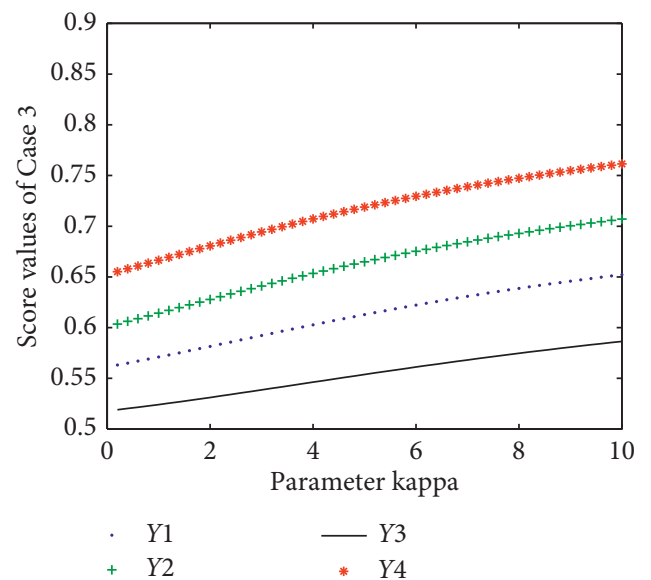

(c)

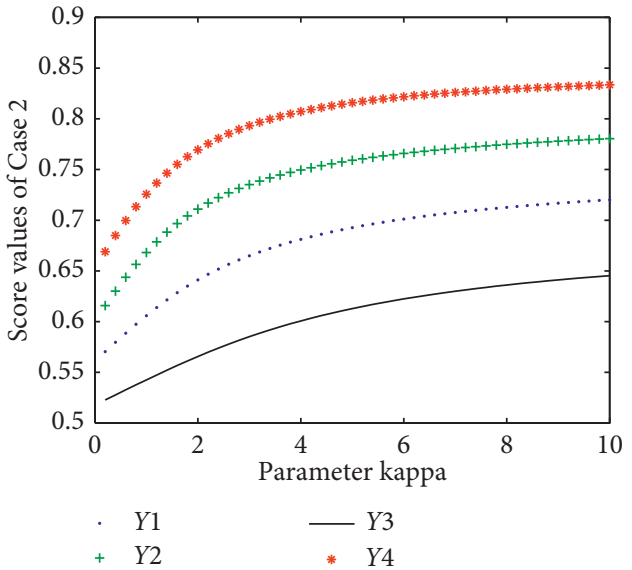

(b)

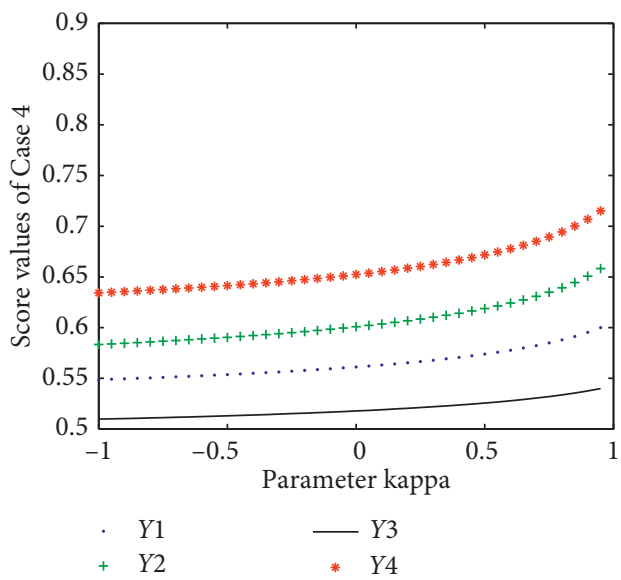

(d)

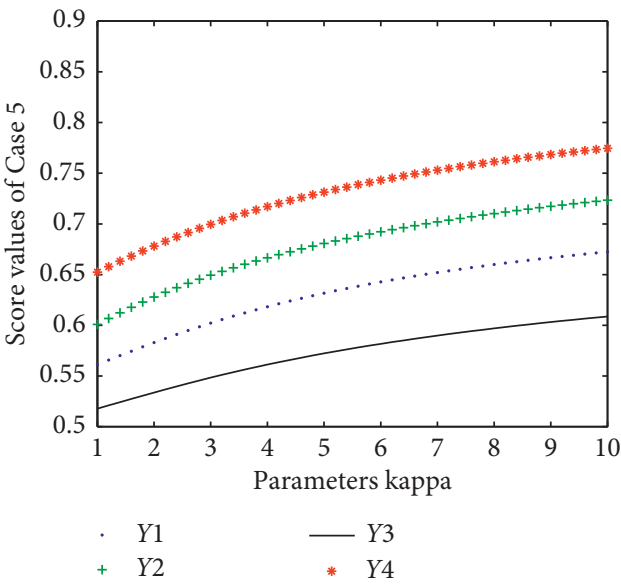

(e)

FIgURe 2: The score functions obtained by AC-HFWA.

which can resize the aggregate value on account of actual decisions, the regularity of our parameter changes is stronger than Zhu's method. The ACHFWA operator has an ideal property of monotonically increasing with respect to the parameter, and the AC-HFWG operator has an ideal property of monotonically decreasing with respect to the parameter, which provides a basis for DMs to select appropriate values according to their risk appetite. If the DM is risk preference, we can choose the largest parameter possible, and if the DM is risk aversion, we can choose the smallest parameter possible. 
TABLE 4: The ordering results obtained by AC-HFWG.

\begin{tabular}{|c|c|c|c|c|c|c|}
\hline Functions & Parameter $\lambda$ & $\mu\left(\Upsilon_{1}\right)$ & $\mu\left(\Upsilon_{2}\right)$ & $\mu\left(\Upsilon_{3}\right)$ & $\mu\left(\Upsilon_{4}\right)$ & Ranking order \\
\hline \multirow{3}{*}{ Case 1} & 1.1 & 0.4687 & 0.4541 & 0.4627 & 0.5042 & $\Upsilon_{4}>\Upsilon_{1}>\Upsilon_{3}>\Upsilon_{2}$ \\
\hline & 2 & 0.4247 & 0.3970 & 0.4358 & 0.4437 & $\Upsilon_{4}>\Upsilon_{3}>\Upsilon_{1}>\Upsilon_{2}$ \\
\hline & 8 & 0.3249 & 0.2964 & 0.3605 & 0.3365 & $\Upsilon_{3}^{4}>\Upsilon_{4}>\Upsilon_{1}>\Upsilon_{2}$ \\
\hline \multirow{3}{*}{ Case 2} & 1 & 0.4319 & 0.4010 & 0.4389 & 0.4573 & $\Upsilon_{4}>\Upsilon_{3}>\Upsilon_{1}>\Upsilon_{2}$ \\
\hline & 2 & 0.3962 & 0.3601 & 0.4159 & 0.4147 & $\Upsilon_{3}>\Upsilon_{4}>\Upsilon_{1}>\Upsilon_{2}$ \\
\hline & 3 & 0.3699 & 0.3346 & 0.3982 & 0.3858 & $\Upsilon_{3}>\Upsilon_{4}>\Upsilon_{1}>\Upsilon_{2}$ \\
\hline \multirow{3}{*}{ Case 3} & 1 & 0.4642 & 0.4482 & 0.4599 & 0.5257 & $\Upsilon_{4}>\Upsilon_{1}>\Upsilon_{3}>\Upsilon_{2}$ \\
\hline & 3 & 0.4417 & 0.4190 & 0.4462 & 0.5212 & $\Upsilon_{4}^{4}>\Upsilon_{3}>\Upsilon_{1}>\Upsilon_{2}$ \\
\hline & 8 & 0.3904 & 0.3628 & 0.4124 & 0.4036 & $\Upsilon_{3}>\Upsilon_{4}>\Upsilon_{1}>\Upsilon_{2}$ \\
\hline \multirow{3}{*}{ Case 4} & -0.9 & 0.4866 & 0.4803 & 0.4732 & 0.5317 & $\Upsilon_{4}>\Upsilon_{1}>\Upsilon_{2}>\Upsilon_{3}$ \\
\hline & 0.25 & 0.4689 & 0.4547 & 0.4627 & 0.5051 & $\Upsilon_{4}>\Upsilon_{1}>\Upsilon_{3}>\Upsilon_{2}$ \\
\hline & 0.75 & 0.4507 & 0.4290 & 0.4511 & 0.4804 & $\Upsilon_{4}>\Upsilon_{3}>\Upsilon_{1}>\Upsilon_{2}$ \\
\hline \multirow{3}{*}{ Case 5} & 1 & 0.4744 & 0.4625 & 0.4661 & 0.5210 & $\Upsilon_{4}>\Upsilon_{1}>\Upsilon_{3}>\Upsilon_{2}$ \\
\hline & 2 & 0.4503 & 0.4295 & 0.4526 & 0.5157 & $\Upsilon_{4}^{4}>\Upsilon_{3}>\Upsilon_{1}>\Upsilon_{2}$ \\
\hline & 8 & 0.3645 & 0.3399 & 0.3922 & 0.3750 & $\Upsilon_{3}>\Upsilon_{4}>\Upsilon_{1}>\Upsilon_{2}$ \\
\hline
\end{tabular}

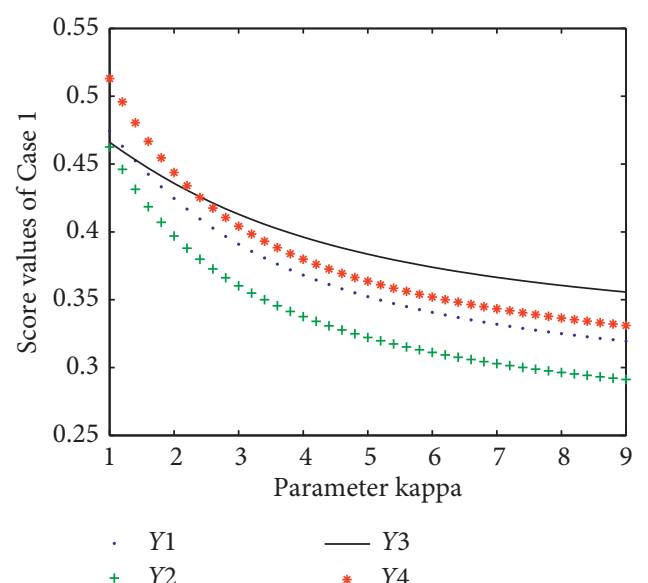

(a)

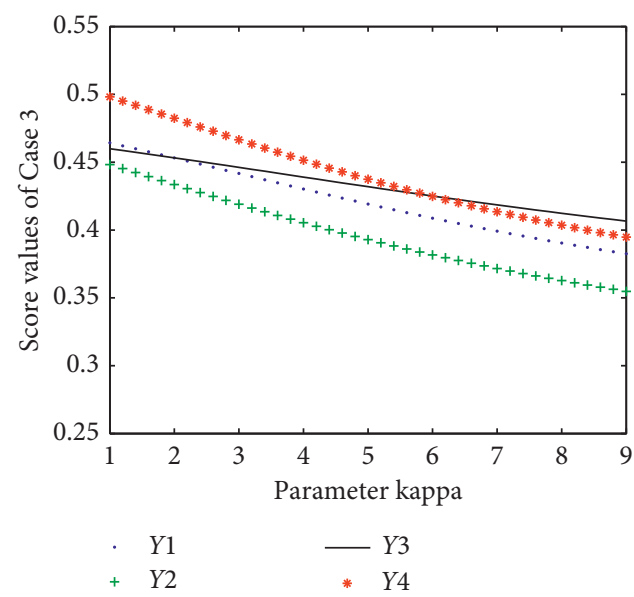

(c)

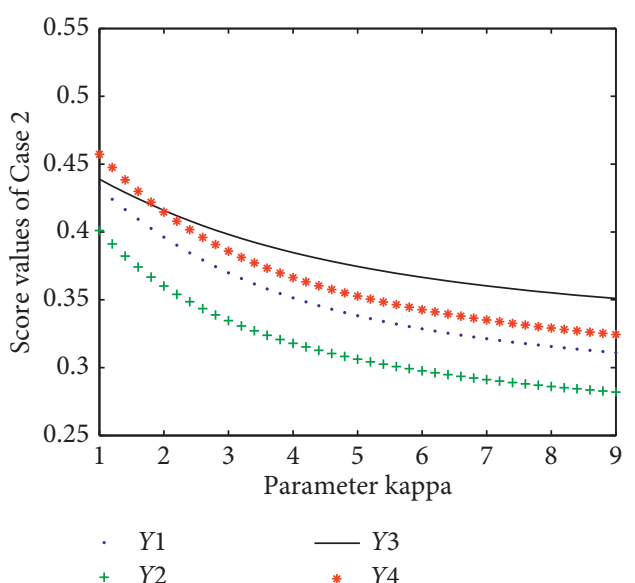

(b)

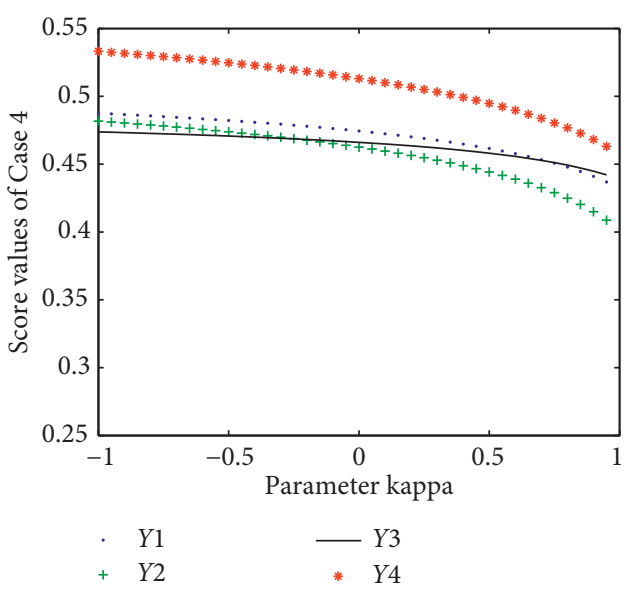

(d)

FIgURE 3: Continued. 


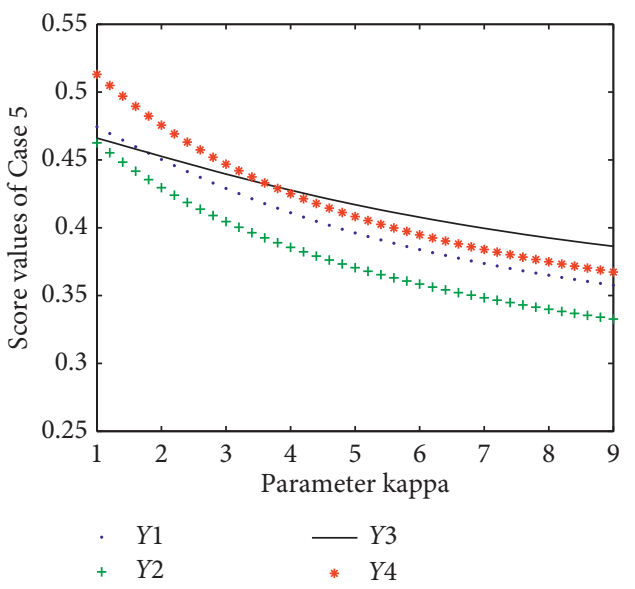

(e)

FIgURE 3: The score functions obtained by AC-HFWG.

TABLE 5: The ordering results obtained by AC-GHFWA in Case 1.

\begin{tabular}{ccccccr}
\hline Parameter $\kappa$ & Parameter $\theta$ & $\mu\left(\Upsilon_{1}\right)$ & $\mu\left(\Upsilon_{2}\right)$ & $\mu\left(\Upsilon_{3}\right)$ & $\mu\left(\Upsilon_{4}\right)$ & Ranking order \\
\hline \multirow{4}{*}{1} & 1 & 0.5612 & 0.6009 & 0.5178 & 0.6524 & $\Upsilon_{4}>\Upsilon_{2}>\Upsilon_{1}>\Upsilon_{3}$ \\
& 5 & 0.6317 & 0.6806 & 0.5722 & 0.7313 & $\Upsilon_{4}>\Upsilon_{2}>\Upsilon_{1}>\Upsilon_{3}$ \\
& 10 & 0.6701 & 0.7236 & 0.6079 & 0.7745 & $\Upsilon_{4}>\Upsilon_{2}>\Upsilon_{1}>\Upsilon_{3}$ \\
\hline & 1 & 0.6757 & 0.7356 & 0.6045 & 0.7893 & $\Upsilon_{4}>\Upsilon_{2}>\Upsilon_{1}>\Upsilon_{3}$ \\
& 5 & 0.6852 & 0.7442 & 0.6142 & 0.7972 & $\Upsilon_{4}>\Upsilon_{2}>\Upsilon_{1}>\Upsilon_{3}$ \\
10 & 10 & 0.6892 & 0.7479 & 0.6186 & 0.8005 & $\Upsilon_{4}>\Upsilon_{2}>\Upsilon_{1}>\Upsilon_{3}$ \\
& 1 & 0.7081 & 0.7688 & 0.6386 & 0.8199 & $\Upsilon_{4}>\Upsilon_{2}>\Upsilon_{1}>\Upsilon_{3}$ \\
& 5 & 0.7112 & 0.7712 & 0.6420 & 0.8219 & $\Upsilon_{4}>\Upsilon_{2}>\Upsilon_{1}>\Upsilon_{3}$ \\
& 10 & 0.7126 & 0.7723 & 0.6435 & 0.8227 & $\Upsilon_{4}>\Upsilon_{2}>\Upsilon_{1}>\Upsilon_{3}$ \\
\hline
\end{tabular}

TABLE 6: The ordering results obtained by AC-GHFWG in Case 1.

\begin{tabular}{ccccccc}
\hline Parameter $\kappa$ & Parameter $\theta$ & $\mu\left(\Upsilon_{1}\right)$ & $\mu\left(\Upsilon_{2}\right)$ & $\mu\left(\Upsilon_{3}\right)$ & $\mu\left(\Upsilon_{4}\right)$ & Ranking order \\
\hline \multirow{1}{*}{1} & 1 & 0.4744 & 0.4625 & 0.4661 & 0.5131 & $\Upsilon_{4}>\Upsilon_{1}>\Upsilon_{3}>\Upsilon_{2}$ \\
& 5 & 0.3962 & 0.3706 & 0.4171 & 0.4083 & $\Upsilon_{3}>\Upsilon_{4}>\Upsilon_{1}>\Upsilon_{2}$ \\
\hline \multirow{3}{*}{5} & 10 & 0.3562 & 0.3262 & 0.3808 & 0.3607 & $\Upsilon_{3}>\Upsilon_{4}>\Upsilon_{1}>\Upsilon_{2}$ \\
& 5 & 0.3523 & 0.3222 & 0.3836 & 0.3636 & $\Upsilon_{3}>\Upsilon_{4}>\Upsilon_{1}>\Upsilon_{2}$ \\
& 10 & 0.3417 & 0.3124 & 0.3746 & 0.3527 & $\Upsilon_{3}>\Upsilon_{4}>\Upsilon_{1}>\Upsilon_{2}$ \\
10 & 1 & 0.3372 & 0.3083 & 0.3706 & 0.3481 & $\Upsilon_{3}>\Upsilon_{4}>\Upsilon_{1}>\Upsilon_{2}$ \\
& 5 & 0.3200 & 0.2870 & 0.3517 & 0.3266 & $\Upsilon_{3}>\Upsilon_{4}>\Upsilon_{1}>\Upsilon_{2}$ \\
& 10 & 0.3165 & 0.2841 & 0.3484 & 0.3234 & $\Upsilon_{3}>\Upsilon_{4}>\Upsilon_{1}>\Upsilon_{2}$ \\
\hline
\end{tabular}

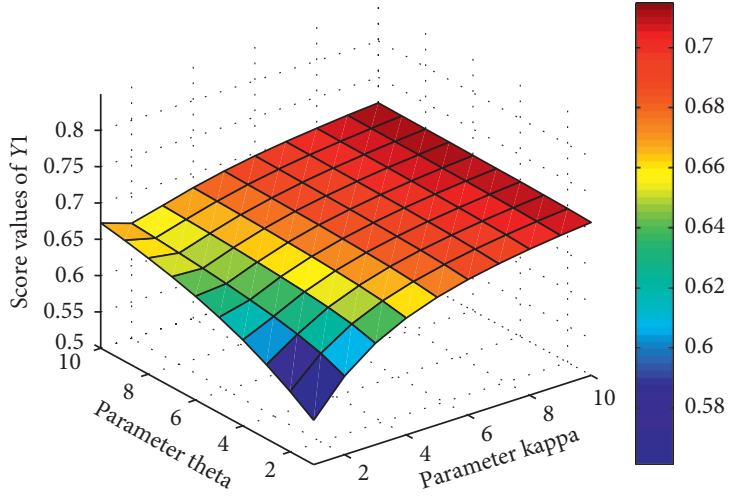

(a)

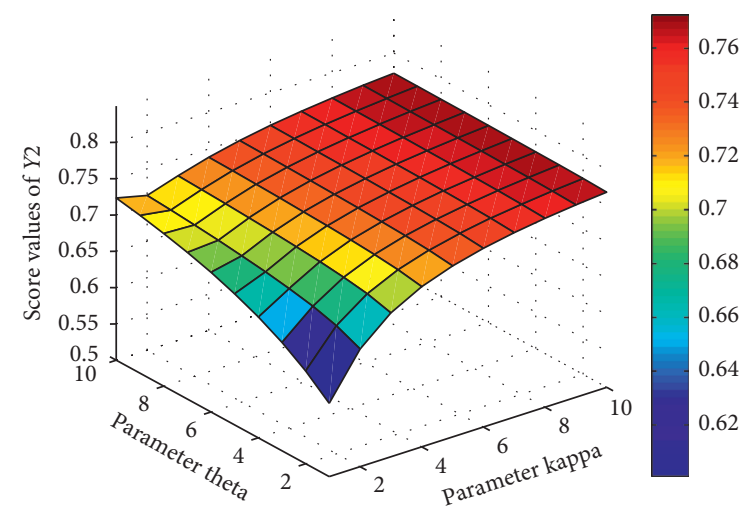

(b)

Figure 4: Continued. 


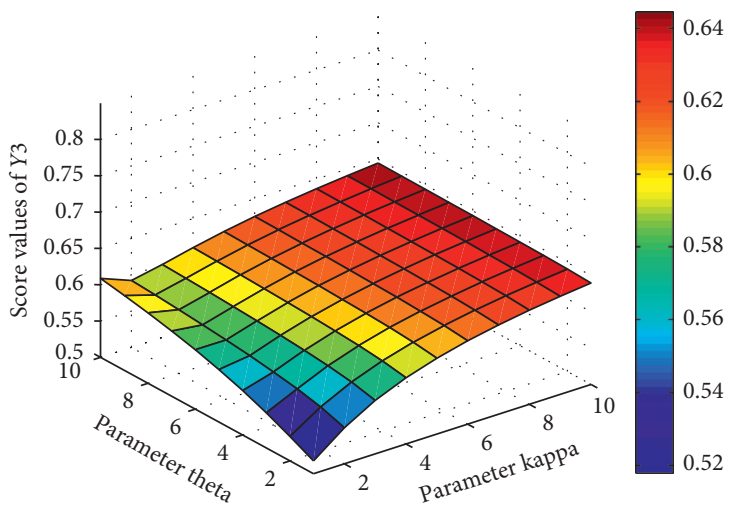

(c)

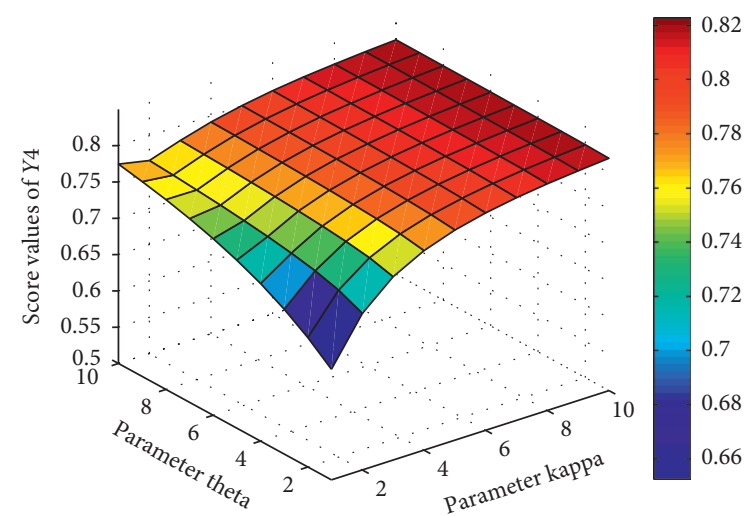

(d)

Figure 4: The score functions obtained by AC-GHFWA in Case 1.

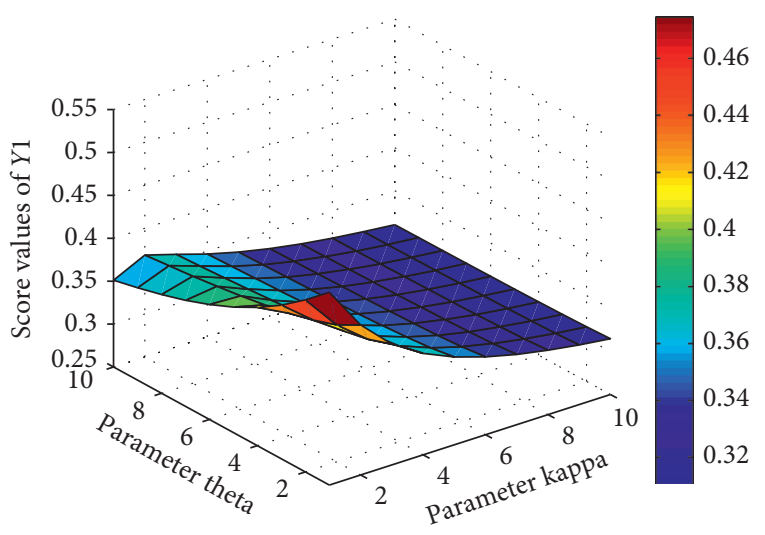

(a)

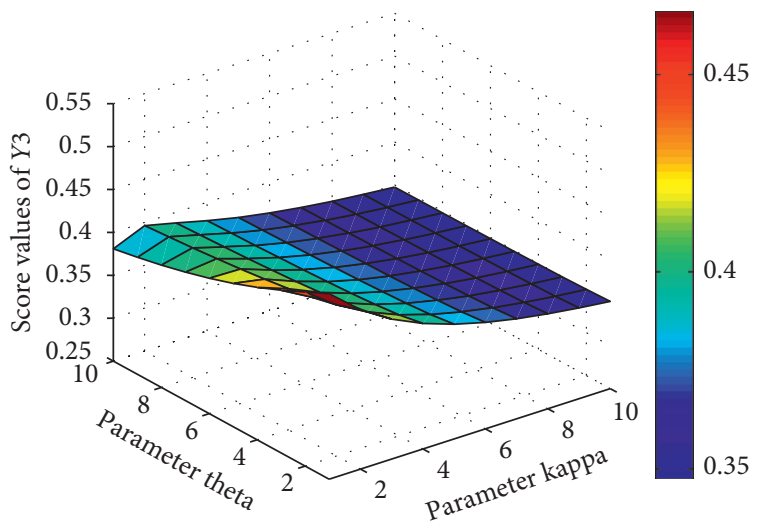

(c)

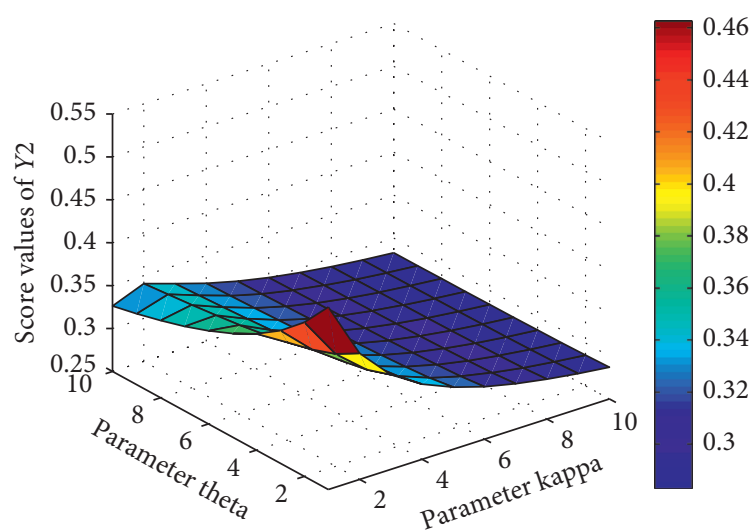

(b)

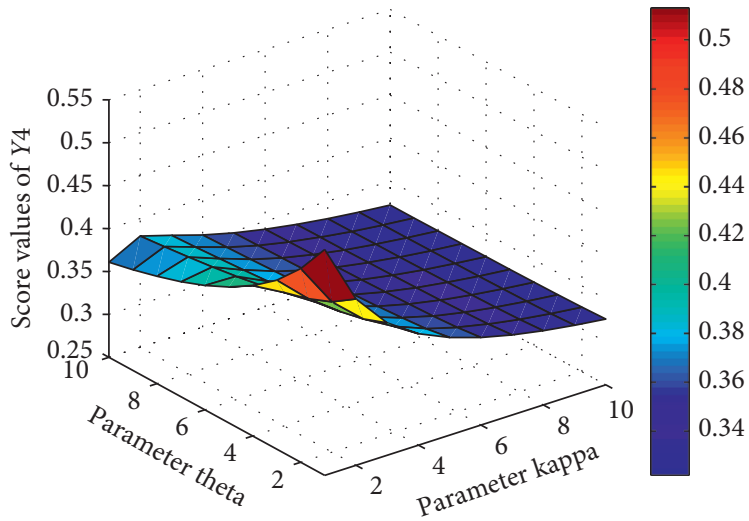

(d)

Figure 5: The score functions obtained by AC-GHFWG in Case 1.

TABLE 7: The scores obtained by different operators.

\begin{tabular}{|c|c|c|c|c|c|c|c|}
\hline Operator & Parameter & $\mu\left(A_{1}\right)$ & $\mu\left(A_{2}\right)$ & $\mu\left(A_{3}\right)$ & $\mu\left(A_{4}\right)$ & $\mu\left(A_{5}\right)$ & $\mu\left(A_{6}\right)$ \\
\hline HFWA [13] & None & 0.4722 & 0.6098 & 0.5988 & 0.4370 & 0.5479 & 0.6969 \\
\hline HFWG [13] & None & 0.4033 & 0.5064 & 0.4896 & 0.3354 & 0.4611 & 0.6472 \\
\hline HFBM [32] & $p=2, q=1$ & 0.5372 & 0.5758 & 0.5576 & 0.3973 & 0.5275 & 0.7072 \\
\hline HFFWA [22] & $\lambda=2$ & 0.3691 & 0.5322 & 0.4782 & 0.3759 & 0.4708 & 0.6031 \\
\hline HFFWG [22] & $\lambda=2$ & 0.5419 & 0.6335 & 0.6232 & 0.4712 & 0.6029 & 0.7475 \\
\hline AC-HFWA (Case 2) & $\lambda=1$ & 0.5069 & 0.6647 & 0.5985 & 0.4916 & 0.5888 & 0.7274 \\
\hline AC-HFWG (Case 2) & $\lambda=1$ & 0.3706 & 0.4616 & 0.4574 & 0.2873 & 0.4158 & 0.6308 \\
\hline
\end{tabular}




\section{Conclusions}

From the above analysis, while copulas and cocopulas are described as different functions with different parameters, there are many different HF information aggregation operators, which can be considered as a reflection of DM's preferences. These operators include specific cases which enable us to select the one that best fits with our interests in respective decision environments. This is the main advantage of these operators. Of course, they also have some shortcomings, which provide us with ideas for the following work.

We will apply the operators to deal with hesitant fuzzy linguistic [33], hesitant Pythagorean fuzzy sets [34], and multiple attribute group decision making [35] in the future. In order to reduce computation, we will also consider to simplify the operation of HFS and redefine the distance between HFEs [36] and score function [37]. Besides, the application of those operators to different decision-making methods will be developed, such as pattern recognition, information retrieval, and data mining.

The operators studied in this paper are based on the known weights. How to use the information of the data itself to determine the weight is also our next work. Liu and Liu [38] studied the generalized intuitional trapezoid fuzzy power averaging operator, which is the basis of our introduction of power mean operators into hesitant fuzzy sets. Wang and Li [39] developed power Bonferroni mean (PBM) operator to integrate Pythagorean fuzzy information, which gives us the inspiration to research new aggregation operators by combining copulas with PBM. Wu et al. [40] extended the best-worst method (BWM) to interval type-2 fuzzy sets, which inspires us to also consider using BWM in more fuzzy environments to determine weights.

\section{Data Availability}

The data used to support the findings of this study are available from the corresponding author upon request.

\section{Conflicts of Interest}

The authors declare that they have no conflicts of interest.

\section{Acknowledgments}

Sichuan Province Youth Science and Technology Innovation Team (No. 2019JDTD0015); The Application Basic Research Plan Project of Sichuan Province (No.2017JY0199); The Scientific Research Project of Department of Education of Sichuan Province (18ZA0273, 15TD0027); The Scientific Research Project of Neijiang Normal University (18TD08); The Scientific Research Project of Neijiang Normal University (No. 16JC09); Open fund of Data Recovery Key Lab of Sichuan Province (No. DRN19018).

\section{References}

[1] L. A. Zadeh, "Fuzzy sets," Information and Control, vol. 8, no. 3, pp. 338-353, 1965.
[2] K. T. Atanassov, "Intuitionistic fuzzy sets," Fuzzy Sets and Systems, vol. 20, no. 1, pp. 87-96, 1986.

[3] K. Atanassov and G. Gargov, "Interval valued intuitionistic fuzzy sets," Fuzzy Sets and Systems, vol. 31, no. 3, pp. 343-349, 1989.

[4] V. Torra and Y. Narukawa, "On hesitant fuzzy sets and decision," in Proceedings of the 2009 IEEE International Conference on Fuzzy Systems, pp. 1378-1382, IEEE, Jeju Island, Republic of Korea, August 2009.

[5] V. Torra, "Hesitant fuzzy sets," International Journal of Intelligent Systems, vol. 25, pp. 529-539, 2010.

[6] R. R. Yager and A. M. Abbasov, "Pythagorean membership grades, complex numbers, and decision making," International Journal of Intelligent Systems, vol. 28, no. 5, pp. 436452, 2013.

[7] R. Sahin, "Cross-entropy measure on interval neutrosophic sets and its applications in multicriteria decision making," Neural Computing and Applications, vol. 28, no. 5, pp. 1177-1187, 2015.

[8] J. Fodor, J.-L. Marichal, M. Roubens, and M. Roubens, "Characterization of the ordered weighted averaging operators," IEEE Transactions on Fuzzy Systems, vol. 3, no. 2, pp. 236-240, 1995.

[9] F. Chiclana, F. Herrera, and E. Herrera-viedma, "The ordered weighted geometric operator: properties and application in MCDM problems," Technologies for Constructing Intelligent Systems, Springer, vol. 2, pp. 173-183, , Berlin, Germany, 2002.

[10] R. R. Yager, "Generalized OWA aggregation operators," Fuzzy Optimization and Decision Making, vol. 3, no. 1, pp. 93-107, 2004.

[11] R. R. Yager and Z. Xu, "The continuous ordered weighted geometric operator and its application to decision making," Fuzzy Sets and Systems, vol. 157, no. 10, pp. 1393-1402, 2006.

[12] J. Merigo and A. Gillafuente, "The induced generalized OWA operator," Information Sciences, vol. 179, no. 6, pp. 729-741, 2009.

[13] M. Xia and Z. Xu, "Hesitant fuzzy information aggregation in decision making," International Journal of Approximate Reasoning, vol. 52, no. 3, pp. 395-407, 2011.

[14] G. Wei, "Hesitant fuzzy prioritized operators and their application to multiple attribute decision making," KnowledgeBased Systems, vol. 31, no. 7, pp. 176-182, 2012.

[15] M. Xia, Z. Xu, and N. Chen, "Some hesitant fuzzy aggregation operators with their application in group decision making," Group Decision and Negotiation, vol. 22, no. 2, pp. 259-279, 2013.

[16] G. Qian, H. Wang, and X. Feng, "Generalized hesitant fuzzy sets and their application in decision support system," Knowledge-Based Systems, vol. 37, no. 4, pp. 357-365, 2013.

[17] Z. Zhang, C. Wang, D. Tian, and K. Li, "Induced generalized hesitant fuzzy operators and their application to multiple attribute group decision making," Computers \& Industrial Engineering, vol. 67, no. 1, pp. 116-138, 2014.

[18] F. Li, X. Chen, and Q. Zhang, "Induced generalized hesitant fuzzy Shapley hybrid operators and their application in multiattribute decision making," Applied Soft Computing, vol. 28, no. 1, pp. 599-607, 2015.

[19] D. Yu, "Some hesitant fuzzy information aggregation operators based on Einstein operational laws," International Journal of Intelligent Systems, vol. 29, no. 4, pp. 320-340, 2014.

[20] R. Lin, X. Zhao, H. Wang, and G. Wei, "Hesitant fuzzy Hamacher aggregation operators and their application to multiple attribute decision making," Journal of Intelligent \& Fuzzy Systems, vol. 27, no. 1, pp. 49-63, 2014. 
[21] Y. Liu, J. Liu, and Y. Qin, "Pythagorean fuzzy linguistic Muirhead mean operators and their applications to multiattribute decision making," International Journal of Intelligent Systems, vol. 35, no. 2, pp. 300-332, 2019.

[22] J. Qin, X. Liu, and W. Pedrycz, "Frank aggregation operators and their application to hesitant fuzzy multiple attribute decision making," Applied Soft Computing, vol. 41, pp. 428452, 2016.

[23] Q. Yu, F. Hou, Y. Zhai, and Y. Du, "Some hesitant fuzzy Einstein aggregation operators and their application to multiple attribute group decision making," International Journal of Intelligent Systems, vol. 31, no. 7, pp. 722-746, 2016.

[24] H. Du, Z. Xu, and F. Cui, "Generalized hesitant fuzzy harmonic mean operators and their applications in group decision making," International Journal of Fuzzy Systems, vol. 18, no. 4, pp. 685-696, 2016.

[25] X. Li and X. Chen, "Generalized hesitant fuzzy harmonic mean operators and their applications in group decision making," Neural Computing and Applications, vol. 31, no. 12, pp. 8917-8929, 2019.

[26] M. Sklar, "Fonctions de rpartition $\mathrm{n}$ dimensions et leurs marges," Universit Paris, vol. 8, pp. 229-231, 1959.

[27] Z. Tao, B. Han, L. Zhou, and H. Chen, "The novel computational model of unbalanced linguistic variables based on archimedean copula," International Journal of Uncertainty, Fuzziness and Knowledge-Based Systems, vol. 26, no. 4, pp. 601-631, 2018.

[28] T. Chen, S. S. He, J. Q. Wang, L. Li, and H. Luo, "Novel operations for linguistic neutrosophic sets on the basis of Archimedean copulas and co-copulas and their application in multi-criteria decision-making problems," Journal of Intelligent and Fuzzy Systems, vol. 37, no. 3, pp. 1-26, 2019.

[29] R. B. Nelsen, "An introduction to copulas," Technometrics, vol. 42 , no. 3 , p. $317,2000$.

[30] C. Genest and R. J. Mackay, "Copules archimédiennes et families de lois bidimensionnelles dont les marges sont données," Canadian Journal of Statistics, vol. 14, no. 2, pp. 145-159, 1986.

[31] U. Cherubini, E. Luciano, and W. Vecchiato, Copula Methods in Finance, John Wiley \& Sons, Hoboken, NJ, USA, 2004.

[32] B. Zhu and Z. S. Xu, "Hesitant fuzzy Bonferroni means for multi-criteria decision making," Journal of the Operational Research Society, vol. 64, no. 12, pp. 1831-1840, 2013.

[33] P. Xiao, Q. Wu, H. Li, L. Zhou, Z. Tao, and J. Liu, "Novel hesitant fuzzy linguistic multi-attribute group decision making method based on improved supplementary regulation and operational laws," IEEE Access, vol. 7, pp. 32922-32940, 2019.

[34] Q. Wu, W. Lin, L. Zhou, Y. Chen, and H. Y. Chen, "Enhancing multiple attribute group decision making flexibility based on information fusion technique and hesitant Pythagorean fuzzy sets," Computers \& Industrial Engineering, vol. 127, pp. 954-970, 2019.

[35] Q. Wu, P. Wu, L. Zhou, H. Chen, and X. Guan, "Some new Hamacher aggregation operators under single-valued neutrosophic 2-tuple linguistic environment and their applications to multi-attribute group decision making," Computers \& Industrial Engineering, vol. 116, pp. 144-162, 2018.

[36] Z. Xu and M. Xia, "Distance and similarity measures for hesitant fuzzy sets," Information Sciences, vol. 181, no. 11, pp. 2128-2138, 2011.

[37] H. Xing, L. Song, and Z. Yang, "An evidential prospect theory framework in hesitant fuzzy multiple-criteria decision-making," Symmetry, vol. 11, no. 12, p. 1467, 2019.
[38] P. Liu and Y. Liu, "An approach to multiple attribute group decision making based on intuitionistic trapezoidal fuzzy power generalized aggregation operator," International Journal of Computational Intelligence Systems, vol. 7, no. 2, pp. 291-304, 2014.

[39] L. Wang and N. Li, "Pythagorean fuzzy interaction power Bonferroni mean aggregation operators in multiple attribute decision making," International Journal of Intelligent Systems, vol. 35, no. 1, pp. 150-183, 2020.

[40] Q. Wu, L. Zhou, Y. Chen, and H. Chen, "An integrated approach to green supplier selection based on the interval type-2 fuzzy best-worst and extended VIKOR methods," Information Sciences, vol. 502, pp. 394-417, 2019. 